\begin{abstract}
IMPROVED MOMENTUM SPREAD FOR PRECISION PHYSICS EXPERIMENTS USING WEDGES
\end{abstract}

\author{
Nick Amato, MS \\ Department of Physics \\ Northern Illinois University, 2019 \\ Michael J. Syphers, Director
}

High precision experiments have become key in the search for physics beyond the Standard Model. One measurement that can be made in order to expand our knowledge of the Standard Model is the precise measurement of the magnetic dipole moment (MDM) of the muon. In order to perform this precise measurement of the MDM of muons, the storage of particles with a precisely known momentum in a highly uniform magnetic field is required.

This thesis is focused on how one may use a wedge of energy absorbing material and the optical parameters of an accelerator lattice to improve upon the amount of particles delivered into a storage ring. This thesis will rely on computer simulations to determine ideal optics parameters as well as to study how the beam parameters change as the beam traverses a lattice. As a case study, this thesis will look to the M4 and M5 lines in the Fermilab Muon $g$-2 experiment, where a wedge of energy absorbing material will be used to reduce the energy spread of a muon beam. 
NORTHERN ILLINOIS UNIVERSITY

DE KALB, ILLINOIS

MAY 2019

\title{
IMPROVED MOMENTUM SPREAD FOR PRECISION PHYSICS EXPERIMENTS USING WEDGES
}

\author{
BY \\ NICK AMATO \\ (C) 2019 Nick Amato
}

A THESIS SUBMITTED TO THE GRADUATE SCHOOL IN PARTIAL FULFILLMENT OF THE REQUIREMENTS

FOR THE DEGREE

MASTER OF SCIENCE

DEPARTMENT OF PHYSICS

Thesis Director:

Michael J. Syphers 


\section{ACKNOWLEDGEMENTS}

I would like to acknowledge Dr. Michael Syphers and Dr. Diktys Stratakis, who offered me assistance and guidance numerous times throughout my research. I would also like to acknowledge the help that Aakaash Narayanan and Prudhvi Raj Varma Chintalapati provided me with while I was getting started learning about accelerator physics and troubleshooting various errors in coding. I would also like to thank my entire thesis de-

fense committee which consisted of Dr. Michael Syphers, Dr. Diktys Stratakis, Dr. Jahred Adelman, and Dr. Phillippe Piot.

This work was supported by the National Science Foundation Grant 1623691. A portion of the work was performed at Fermi National Accelerator Laboratory which is operated by the Fermi Research Alliance, LLC under Contract No. DE-AC02-07CH11359 with the United States Department of Energy. 


\section{DEDICATION}

I dedicate this work to my parents for supporting me throughout my entire educational career. 


\section{TABLE OF CONTENTS}

List of Figures $\ldots \ldots \ldots \ldots \ldots \ldots \ldots \ldots \ldots \ldots \ldots \ldots \ldots \ldots \ldots$ vii

\section{Chapter}

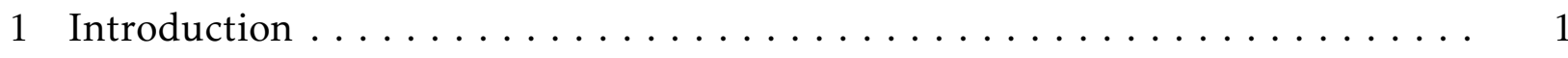

1.1 Magnetic and Electric Dipole Moment . . . . . . . . . . . . . . 2

1.2 Beam Physics Concepts ....................... 3

1.2.1 Transverse Coordinates ..................... 3

1.2.2 Matrix Formalism ...................... 8

1.2.3 Quadrupoles and Thin Lenses ..................... 11

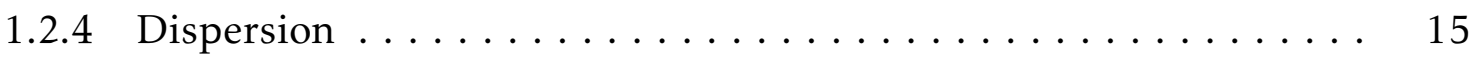

1.2.5 Ellipses and Emittance. . . . . . . . . . . . . . . . 16

1.2.5.1 Algebraic Formalism . . . . . . . . . . . . 17

1.2.5.2 Matrix Formalism ................... 18

1.2.5.3 Sigma Matrix. . . . . . . . . . . . . . . . 19

1.2.5.4 Emittance ....................... 20

2 Beam Interactions With Matter. . . . . . . . . . . . . . . . . . 22

2.1 Momentum Spread Reduction . . . . . . . . . . . . . . . 22

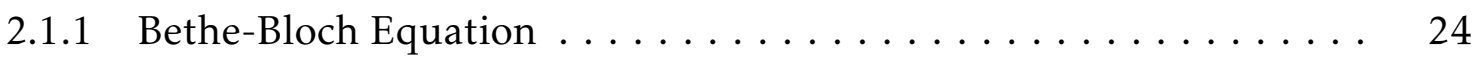

2.2 Emittance Growth . . . . . . . . . . . . . . . . . . 26

2.2.1 Coulomb Scattering. . . . . . . . . . . . . . . . . 26

2.2.2 Wedge Consideration. . . . . . . . . . . . . . . . . 27 
Chapter Page

3 The Fermilab Muon Beam . . . . . . . . . . . . . . . . . . . . . 28

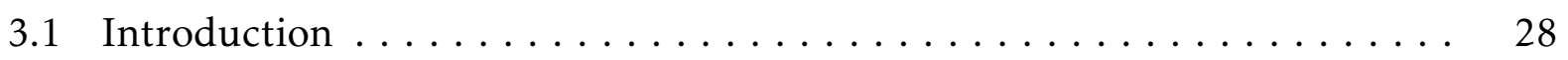

3.2 Beam Formation and Transport. . . . . . . . . . . . . 28

3.3 The Use of Wedges . . . . . . . . . . . . . . . . . . 31

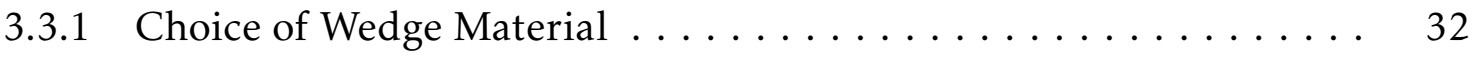

3.3.2 Need For Dispersion . . . . . . . . . . . . . . . . 33

3.3.3 How a Wedge Might Help ... . . . . . . . . . . . . . 33

3.3.4 Ideal Parameters Before The Wedges. . . . . . . . . . . . . . . 34

3.3.5 Recapturing The Beam ...................... 34

3.4 Wedge Position .......................... 34

3.5 Wedge Specifications . . . . . . . . . . . . . . . . . 36

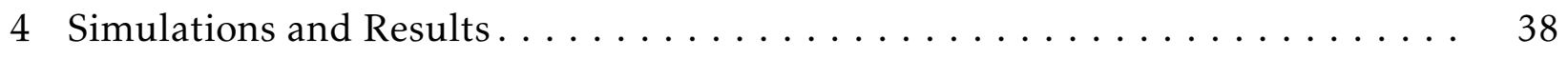

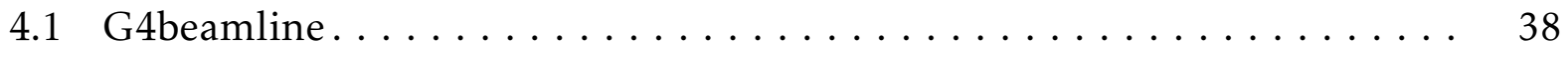

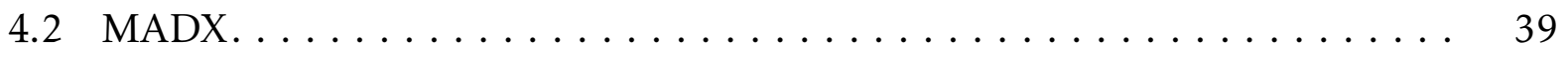

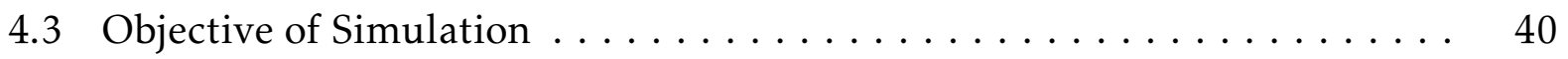

4.4 Ideal Beam Parameters. . . . . . . . . . . . . . . . . 40

4.5 Custom Beam Parameters Simulation . . . . . . . . . . . . . . 41

4.5 .1 Analysis ......................... 42

4.5 .2 Base Distribution ....................... 44

4.5 .3 Modifying Values ......................... 44

4.5.4 Modifying the Base Distribution. . . . . . . . . . . . 46

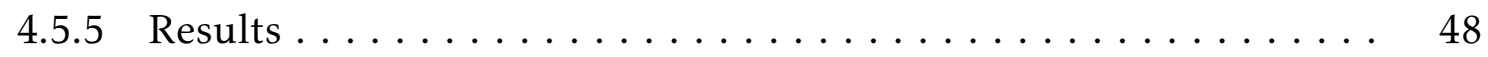

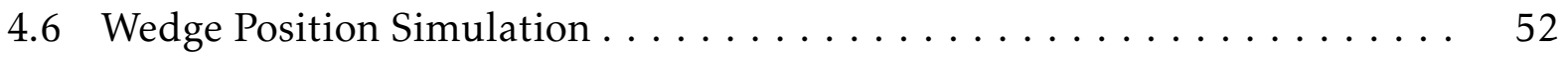

$4.6 .1 \quad$ Analysis ........................... 52 
Chapter

4.6 .2 Results.......................... 54

4.6.2.1 Wedge Effectiveness. . . . . . . . . . . . . . 58

4.7 Wedge Rotation Simulation $\ldots \ldots \ldots \ldots \ldots \ldots \ldots$

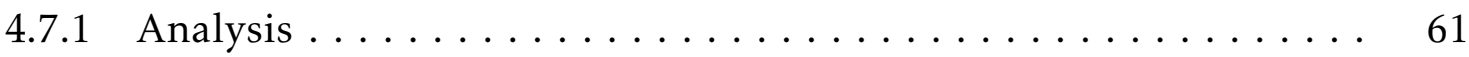

4.7 .2 Results............................. 62

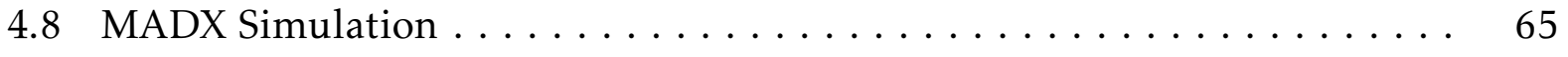

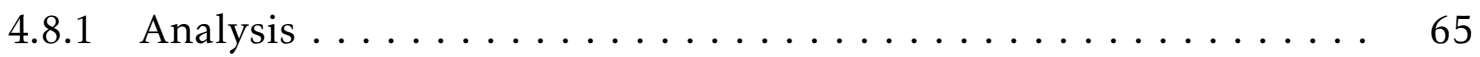

4.8 .2 Results........................ 66

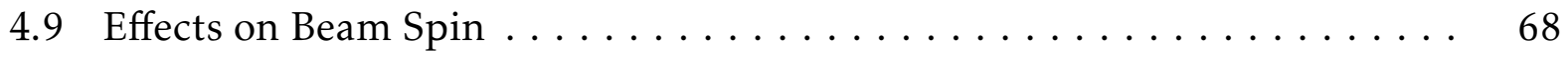

$4.9 .1 \quad$ Analysis .......................... 68

4.9 .2 Results............................ 70

5 Conclusions. .............................. 72

References ............................... 74 


\section{LIST OF FIGURES}

Figure

Page

1.1 Here, a right handed coordinate system is shown where $\hat{s}$ serves as the direction of the particle beam trajectory while $\hat{x}$ and $\hat{y}$ serve as the trans-

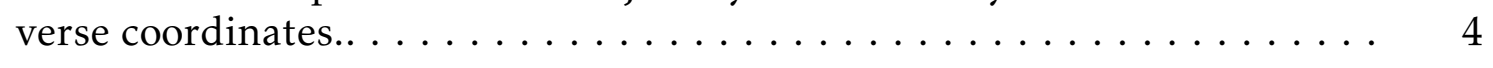

1.2 Here, it is observed how $r$ is defined in terms of $\rho$ and $x . \ldots \ldots$

1.3 Here one observes the theoretical gradient of a magnet. The more a particle strays from the ideal trajectory, the larger the kick it will recieve. 12

1.4 Here one two quadrupoles and a positively charged particle traveling through them. The first quadrupole the particle travels through is focusing in the horizontal plane, while the second is defocusing in the horizontal plane. The black lines in this figure represent the direction of magnetic field, while the red lines represent the direction of the force experienced by the particle traveling through the quad. The direction of the force received by the particle can be found by using the right hand rule.. . 13

1.5 Here one observes the deflection of a particle by a thin lens magnetic

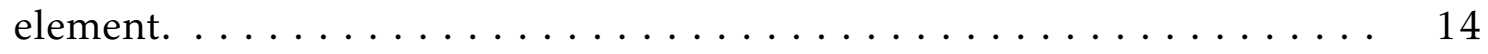

1.6 Here one observes a ray initially parallel to the optical axis. This ray is bent towards the optical axis, through the use of an optical thin lens, which causes the ray to pass through the focal point $f . \ldots \ldots \ldots \ldots$

2.1 The black line represents a muon beam with a $1.2 \% \mathrm{rms}$ width while the blue line represents a muon beam with a $0.6 \%$ rms width. The vertical red lines represent the $0.2 \%$ acceptance range of the the storage ring in

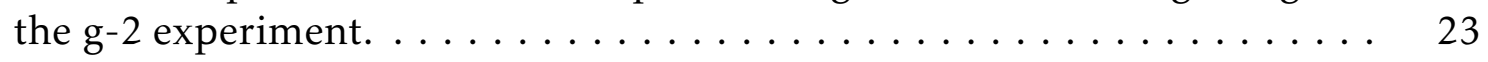

2.2 A particle beam obtains a non-zero dispersion value through the use of dipole bending magnets. A wedge of energy absorbing material is placed in the beams path. ...................... 25 
3.1 Here one observes the series of beamlines necessary to deliver muons to the g-2 storage ring. . . . . . . . . . . . . . . . . 29

3.2 Figure (a) represents the $\beta$ and $D$ values in each transverse coordinate through the entire M5 line. Figure (b) represents a zoomed in view of the M5 line at a point where there is non-zero $D$ values in each transverse coordinate. Both of these figures are with the wedges removed. . . . . . . 35

3.3 Here one observes the series of beamlines necessary to deliver muons to

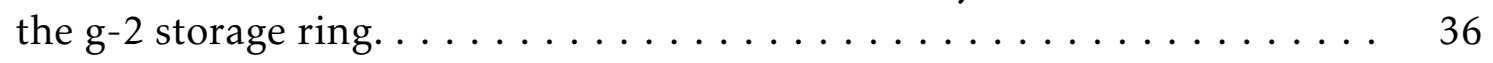

3.4 Figure (a) shows the top view of each wedge while figure (b) shows the height and width of each wedge. $\ldots \ldots \ldots \ldots \ldots \ldots \ldots \ldots \ldots$

3.5 Here, one observes a picture of one of the actual polyethylene wedges to be used in the Fermilab Muon g-2 experiment. $\ldots \ldots \ldots \ldots . \ldots \ldots$

4.1 Here one observes the base distribution passing through the first virtual detector and both wedges on it's way to the observation point. The observation point is itself another virtual detector in G4beamline. . . . . . . . . 42

4.2 Here one observes the generated input distribution traversing through both wedges and to the observation point. . . . . . . . . . . . . 44

4.3 Figure (a) has $\alpha$ focused to zero at the first wedge, while (b) is focused at the dipole between the wedges and (c) is focused at the second wedge. . . . 46

4.4 The captions "case $(1,2,3)$ " refer to the three different distances of $d$ at which $\beta^{*}$ values are focused. Cases 1 and 3 refer to a focal point inside the first and second wedges respectively. Case 2 refers to a focal point inside of the dipole in-between the two wedges. The vertical axis in the images represents the changing $\beta_{\mathrm{y}}$ value. The horizontal axis represents the changing of $\beta_{x}$, while the colored tiles represent $\varepsilon_{y}$ and $\varepsilon_{x}$ values re-

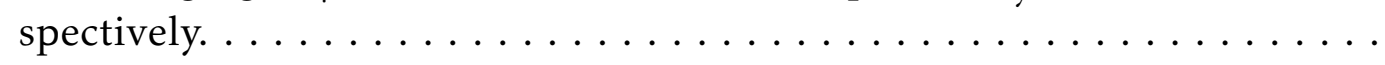

4.5 In this figure, one observes the effect of having a low $\beta_{\mathrm{x}}^{*}$ value of $1 \mathrm{~m}$ at

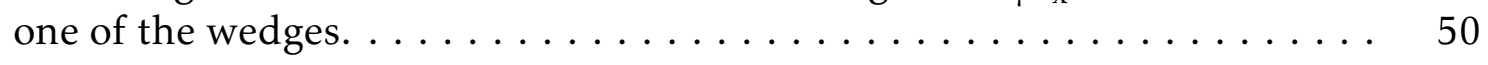

4.6 In this figure, one can observe the optimized percent reduction in $\varepsilon_{\mathrm{x}, \mathrm{y}}$ growth for each of the three cases when compared to the base distribu-

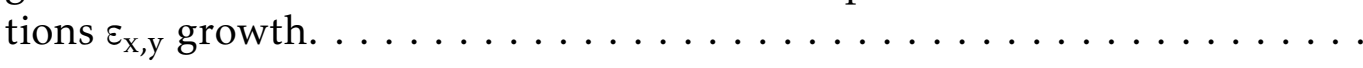


Figure

4.7 This figure represents the standard deviation in momentum of the beam bunch. The data in this plot are taken immediately after the second wedge

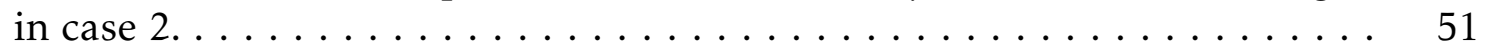

4.8 Moving the wedge into or out of the beam by $20 \mathrm{~mm}$ is represented by the dashed triangle while the solid triangle represents the original position of the wedge. ........................ 53

4.9 In this figure one sees how moving one wedge, with the other removed, affects the amount of particles within the $0.2 \%$ momentum acceptance window at the end of the M5 line. . . . . . . . . . . . 55

4.10 In this figure one observes a scenario in which both wedges are inserted into the lattice and are granted the freedom to move from $-10 \mathrm{~mm}$ out of the beam path to $+20 \mathrm{~mm}$ into the beam path. Here the X axis represents Wedge 2 and the $\mathrm{Y}$ axis represents Wedge 1. This figure displays the amount of particles within the $0.2 \%$ momentum acceptance window at the end of the M5 line scaled to a run with no wedges in place. . . . . . . 56

4.11 Both (a) and (b) represent the emittance, $\varepsilon$, at the end of the M5 line the position of wedge 1 and wedge 2 are altered. . . . . . . . . . . . 58

4.12 The dotted lines in this figure represent the position of the first and sec-

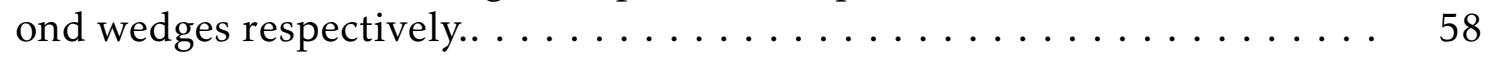

4.13 This figure represents a distribution with small dispersion and a distribution with large dispersion. $\ldots \ldots \ldots \ldots \ldots \ldots \ldots \ldots$

4.14 This figure represents a distribution with large $\beta$ and small $\beta$. . . . . . . . 59

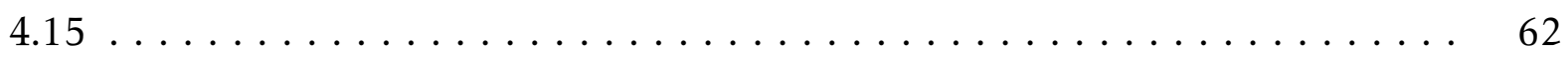

4.16 Here one observes the effects of altering the first wedge's rotation angle on the amount of particles within the momentum acceptance range at the end of the M5 line. This plot is scaled to the amount of particles within the $0.2 \%$ momentum acceptance window at the end of M5 when wedge 1 is at it's original position and rotation angle, $0 \mathrm{~mm}$ and 0deg respectively. . 63

4.17 Here one observes the effects of altering the second wedge's rotation angle on the amount of particles within the momentum acceptance range at the end of the M5 line. This plot is scaled to the amount of particles within the $0.2 \%$ momentum acceptance window at the end of M5 when wedge 2 is at it's original position and rotation angle, $0 \mathrm{~mm}$ and 0 deg respectively..$~ 64$ 
Figure

4.18 Figure (a) represents Case 1, where there are no wedges placed in the lattice. Figure (b) represents Case 2, where both wedges are placed in the lattice at their unaltered position and rotation angle. Each of these figures begins immediately after the second wedge and ends at the end of M5.. . . 66

4.19 This figure displays the differences in $\beta_{x, y}$ and $D_{x, y}$ between Case 1 and

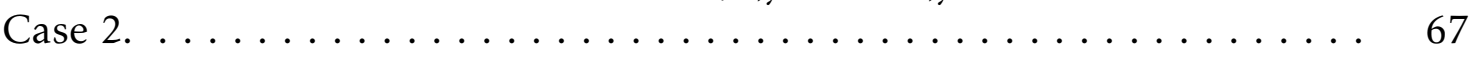

4.20 One simulation will be run with wedges in the lattice, (a), and compared to a run with the wedges removed from the lattice, (b). The observation point is where the spin in each of these two cases will be examined. . . . . 68

4.21 This figure shows how $\phi_{x}$ and $\phi_{y}$ are defined. . . . . . . . . . . . 69

4.22 This figure shows the differences in $\phi_{x}$ and $\phi_{y}$ for a simulation with wedges in the lattice compared to a simulation with the wedges removed

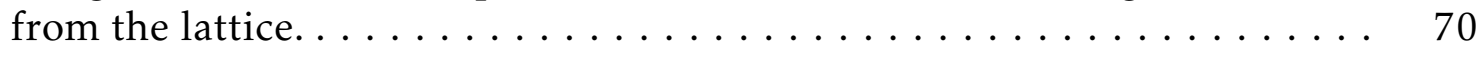




\section{CHAPTER 1 INTRODUCTION}

In order to continue the search for physics beyond the Standard Model, experiments are required to be performed at high precision. Two measurements that will be found most useful in the hunt for new physics are those of the Magnetic Dipole Moment (MDM) and the Electric Dipole Moment (EDM) of the muon.

To measure the magnetic dipole moment of a muon, one requires a muon beam to be circulating in a storage ring. To get optimal results, one wants to store as many muons as possible in this storage ring. the Fermilab Muon g-2 experiment utilizes muons which are produced through the decay of pions. These muons, that are a result of decaying pions, initially have a large momentum spread $\Delta \mathrm{P} / \mathrm{P}$. The storage ring only accepts particles within a certain momentum acceptance window. In order to fit more particles within this momentum acceptance window, one requires a smaller spread in momentum or $\Delta \mathrm{P} / \mathrm{P}$. To reduce the momentum spread of a distribution, one may utilize the technique of passing a beam distribution through an energy absorbing material. In the case of the Fermilab Muon g-2 experiment, the energy absorbing material used will be in the shape of a wedge. The goal, in using an energy absorbing wedge, is to achieve more muons within the momentum acceptance window. If one succeeds in the goal of obtaining more muons within this momentum acceptance window, one will in turn store more muons to be used for experimentation.

One may question the importance of simply storing more muons for an experiment. Take for example an experiment that is proposed to run for a number of years. Now consider that through the use of a wedge absorbing material, the momentum spread $\Delta \mathrm{P} / \mathrm{P}$ 
is reduced and twice as many muons are stored. Given that the experiment has been supplied with twice as many muons as originally thought possible, the experiment's run time can now potentially be cut in half. For this reason, the use of wedges can be seen as an attractive and cost efficient way to improve upon such an experiment.

Through the use of particle tracking software, one will be able to observe how beam parameters are changed as the muon beam traverses the energy absorbing wedges. One will also find it useful to study how the wedge position affects the outcome of stored muons.

\subsection{Magnetic and Electric Dipole Moment}

The magnetic moment is a quantity that is used to represent the magnetic strength and orientation of a magnet, or in this case particle, that creates a magnetic field. Elementary particles have a property called "spin" that manifests itself in the way it is affected by a magnetic field. Through studying how a particle's spin is affected by a magnetic field, one may obtain the magnetic moment of that particle. One such example of a tangible object with a magnetic moment would be a current carrying loop. One can imagine that the muon is a rotating sphere of charge, which is acting like a current carrying loop, and therefore creating a dipole moment. This is a useful analogy, but it is important to note that these muons are not actually "spinning" in the sense of rotation. In order to measure the magnetic dipole moment, one simply needs to observe how the muon interacts with an external field.

The term "magnetic dipole moment" refers to the component of the magnetic moment that can be equivalently represented by a magnetic dipole. The magnetic dipole moment

of the muon has been most recently measured by Brookhaven National Lab (BNL) [1]. 
The result that BNL obtained did not agree with what the standard model predicts the magnetic dipole moment should be, differing by 2.7 standard deviations. In order to confirm the findings of BNL, Fermilab is running the "muon g-2 experiment", which will acquire the magnetic dipole moment of a muon to higher precision. The Fermilab Muon g-2 experiment will measure to a precision of 0.14 parts per million, while BNL measured to a precision of 0.5 parts per million. This measurement of the magnetic dipole moment of a muon could imply that there are more particles to be discovered and that the standard model is not yet complete.

The electric dipole moment is the measurement of the separation of positive and negative electrical charges. In a muon, the presence of an electric dipole moment would be indicative of smaller particles within. Therefore, a non-zero measurement of the EDM in a muon could lead to the expansion of the Standard Model. This paper will not examine the EDM in depth; for more on this topic see [2].

\subsection{Beam Physics Concepts}

\subsubsection{Transverse Coordinates}

Magnetic and electric elements serve as the driving force as well as the guiding force for particles traversing a lattice. In order to fully understand how a particle beam traverses a series of magnetic and electric elements, it is necessary to examine the transverse coordinate plane as in figure 1.1 .

Particle accelerators have apertures that serve as the path for the particles. If the beam was to become unstable, or if the emittance in the transverse plane were to grow too large, one would lose particles to collisions with the beam pipe walls. 


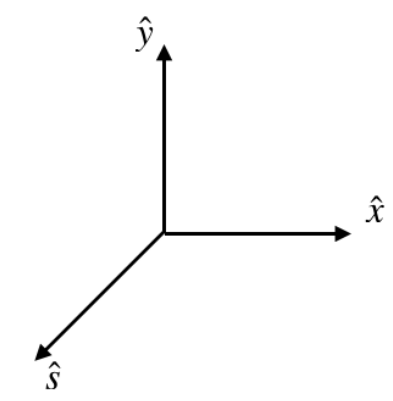

Figure 1.1: Here, a right handed coordinate system is shown where ŝ serves as the direction of the particle beam trajectory while $\hat{x}$ and $\hat{y}$ serve as the transverse coordinates.

It is possible to bound the beam in an oscillatory motion about the design trajectory. This motion is called betatron oscillation. Since the particles transverse oscillation frequencies are much higher than the longitudinal oscillation frequencies, the longitudinal degree of freedom can be treated independently. Ideally, the restoring forces in the two transverse degrees of freedom can also be treated independently.

To examine the particle's equation of motion, one may start with the Lorentz Force Law,

$$
\vec{F}=q \vec{E}+q \vec{v} \times \vec{B}
$$

In the Lorentz Force equation $E$ and $B$ represent the electric and magnetic fields respectively while $q$ represents the charge of the particles traversing these fields.

Now, one can imagine a particle traveling in the $\hat{s}$ direction with $\hat{x}$ and $\hat{y}$ components. Let's say that one wishes to study the $\hat{x}$ component of this particle's motion. Assuming there is no B component in the $\hat{s}$ or $\hat{x}$ direction, the Lorentz force equation now becomes,

$$
\gamma m\left(\ddot{r}-r \dot{\theta}^{2}\right)=\left(-q v_{s} B_{y}\right)
$$


In equation 1.2, the radial position of a particle, $r$, can be written as the radius of curvature of the ideal particle $\rho$ plus the $x$ position relative to this position. the value $m$ corresponds to the mass of the particle being studied while $\gamma$ is the relativistic $\gamma$ as shown in equation 1.4 .

$$
\begin{gathered}
r=\rho+x \\
\gamma=\frac{1}{\sqrt{1-\frac{v^{2}}{c^{2}}}}
\end{gathered}
$$

The relationship between angular and linear velocity is also noted in equation 1.2 ,

$$
v_{s}=r \dot{\theta}
$$

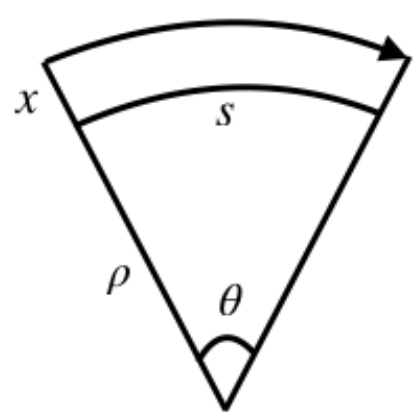

Figure 1.2: Here, it is observed how $r$ is defined in terms of $\rho$ and $x$.

One observes that on the right hand side of equation 1.2 , only the velocity in the $\hat{s}$ and the $\mathrm{B}$ field in the $\hat{y}$ directions survive. This is explained through the use of the right hand rule. In order for a cross product to produce a result in the $\hat{x}$ direction, one must have a cross product between two components that are perpendicular to the $\hat{x}$ direction.

At this time, the equation is time dependent. It will be found useful to change $s$ into the independent variable. 


$$
\frac{d}{d t}=\frac{d s}{d t} \frac{d}{d s}
$$

After applying this identity, one obtains an equation of motion in the $\hat{x}$ direction of the form

$$
\frac{d^{2} x}{d s^{2}}+\left[\frac{1}{\rho^{2}}+\frac{1}{(B \rho)} \frac{\partial B_{y}(s)}{\partial x}\right] x=0
$$

In equation $1.7, B_{\mathrm{y}}$ represents the $\hat{y}$ component of the magnetic field while $(B \rho)$ represents the magnetic rigidity. The value $(B \rho)$ is the ratio of a particle's momentum to it's charge.

$$
(B \rho)=\frac{p}{q}
$$

After a brief inspection of the new found equation of motion, equation 1.7 , one can see that the equation has the form of a simple harmonic oscillator.

$$
x^{\prime \prime}+K(s) x=0
$$

One important thing to keep in mind about this equation, is that instead of $K$ being the "spring constant", it is a function of position $s$. This equation is Hill's equation, a differential equation with solutions that closely resemble that of simple harmonic oscillations.

To find a solution to this equation, it is useful to keep in mind that in accelerators, $K$ is periodic. This is true if it is assumed the accelerator has been designed as a simple quadrupole/dipole system with ideal linear fields. This means that one can write $K(s)=$ $K(s+C)$, where $C$ is some repeat distance. One may assume a solution of the form, 


$$
x=A w(s) \cos [\psi(s)+\delta]
$$

where $A$ and $\delta$ are just some constants reflecting initial conditions and $w(s)$ can be required to be a periodic function. $\varphi$ in this equation represents the phase advance of the particle's oscillatory behavior. Plugging this equation into equation 1.9 , one obtains,

$$
x^{\prime \prime}+K x=A\left(2 w^{\prime} \psi^{\prime}+w \psi^{\prime \prime}\right) \sin (\psi+\delta)+A\left(w^{\prime \prime}-w\left(\psi^{\prime}\right)^{2}+K w\right) \cos (\psi+\delta)=0
$$

Since both functions of $\sin$ and $\cos$ will not vanish through any combination of $\psi+\delta$, their coefficients must be made to equal zero. If one multiplies the $\sin$ term by $w$ one obtains

$$
2 w w^{\prime} \phi^{\prime}+w^{2} \phi^{\prime \prime}=\left(w^{2} \phi^{\prime}\right)^{\prime}=0
$$

or

$$
w(s)^{2} \psi^{\prime}=k
$$

where $k$ is an arbitrary constant of integration.

At this point, a matrix is able to be created to describe how a particles position $x$ and velocity $x^{\prime}$ will change as the particle traverses through the a system of magnetic elements, sometimes referred to as a "lattice". 


\subsubsection{Matrix Formalism}

In order to write matrices that describe how a particle's motion in the $x$ direction changes as it traverses the lattice, equation 1.10 can be re-written as,

$$
x=w(s)\left(A_{1} \cos \psi+A_{2} \sin \psi\right)
$$

and, by differentiating and using equation 1.13,

$$
x^{\prime}=\left(A_{1} w^{\prime}+\frac{A_{2} k}{w}\right) \cos \psi+\left(A_{2} w^{\prime}-\frac{A_{1} k}{w} \sin \psi\right) .
$$

then for the initial conditions $x_{0}, x_{0}{ }^{\prime}$, at $s=s_{0}$, the constants $A_{1}$ and $A_{2}$ are

$$
\begin{gathered}
A_{1}=\frac{x_{0}}{w}, \\
A_{2}=\frac{x_{0}^{\prime} w-x_{0} w^{\prime}}{k} .
\end{gathered}
$$

By requiring that the function $w$ be periodic over the distance $C$, one can now write down the matrix for propagation from $s_{0}$ to $s_{0}+C$. The resulting matrix to describe this motion is

$$
\left[\begin{array}{c}
x \\
x^{\prime}
\end{array}\right]_{s_{0}+C}=\left[\begin{array}{cc}
\cos \Delta \psi-\frac{w w^{\prime}}{k} \sin \Delta \psi & \frac{w^{2}}{k} \sin \Delta \psi \\
\frac{1+\left(w w^{\prime} / k\right)^{2}}{w^{2} / k} \sin \Delta \psi & \cos \Delta \psi+\frac{w w^{\prime}}{k} \sin \Delta \psi
\end{array}\right]\left[\begin{array}{c}
x \\
x^{\prime}
\end{array}\right]_{s_{0}}
$$

One can represent $\varphi$ the phase of the particle's oscillation as it advances through the repeat distance $C$ by the amount 


$$
\psi\left(s_{0}->s_{0}+C\right)=\Delta \psi_{C}=\int_{s_{0}}^{s_{0}+C} \frac{k d s}{w^{2}(s)}
$$

This matrix formalism is indeed very handy, but one can yet put it in a more convenient form. It is useful here to define these matrix in terms of the more fundamental quantities of the problem. Upon inspection of equation 1.18, one notices that the function $w^{2}(\mathrm{~s})$ and its derivative both scale with the arbitrary constant $k$. Since the motion of the particle, and the phase advance of the particle, is what's observed, choosing a different value of $k$ will lead to a different value for the function $w^{2}(\mathrm{~s})$, scaled by a factor of $k$. since $w^{2}(\mathrm{~s})$ and its derivative are the more fundamental quantities of the problem, one may define the new variables

$$
\begin{gathered}
\beta(s) \equiv \frac{w^{2}(s)}{k}, \\
\alpha(s) \equiv-\frac{1}{2} \frac{d \beta(s)}{d s}=-\frac{1}{2} \frac{d}{d s}\left(\frac{w^{2}(s)}{k}\right), \\
\gamma \equiv \frac{1+\alpha^{2}}{\beta} .
\end{gathered}
$$

These new variables as a whole are called the Courant-Snyder parameters, or sometimes Twiss parameters. Along with the introduction of these new variables, one may rewrite the matrix in equation 1.18 as

$$
\left[\begin{array}{c}
x \\
x^{\prime}
\end{array}\right]_{s_{0}+C}=\left[\begin{array}{cc}
\cos \Delta \psi_{C}+\alpha \sin \Delta \psi_{C} & \beta \sin \Delta \psi_{C} \\
-\gamma \sin \Delta \psi_{C} & \cos \Delta \psi_{C}-\alpha \sin \Delta \psi_{C}
\end{array}\right]\left[\begin{array}{l}
x \\
x^{\prime}
\end{array}\right]_{S_{0}}
$$

The phase advance now becomes

$$
\Delta \psi_{C}=\int_{s_{0}}^{s_{0}+C} \frac{d s}{\beta(s)},
$$


A matrix that can tell the new $x$ and $x^{\prime}$ of a particle that has traversed through a periodic section of a lattice has now been obtained, as long it's initial $x$ and $x$ ' are known as well as the phase advance of the location one wishes to observe.

Now, imagine that one is working with a single pass system, in which the particles will only traverse lattice elements once before being transported elsewhere. One will find it useful to continue with the periodic system and re-write matrix 1.18 as

$$
M=I \cos \Delta \psi_{C}+J \sin \Delta \psi_{C}
$$

where

$$
J=\left[\begin{array}{cc}
\alpha & \beta \\
-\gamma & -\alpha
\end{array}\right]
$$

and $I$ is the identity matrix.

Suppose that multiplying all of the individual matrices of some repeat period gives the matrix

$$
M=\left[\begin{array}{ll}
a & b \\
c & d
\end{array}\right]
$$

Now by equating matrix 1.27 and matrix 1.23 , one can arrive at new definitions of the Courant-Snyder parameters.

$$
\begin{gathered}
\beta=\frac{b}{\sin \Delta \psi_{C}} \\
\alpha=\frac{a-d}{2 \sin \Delta \psi_{C}}
\end{gathered}
$$


These two equations now provide the Courant-Snyder parameters at one point throughout the periodic lattice. Recalling that one may wish to examine a single pass system, it is noted that this same procedure works between any pair of corresponding points of the lattice. This allows one to find $\beta(s)$ at all $s$ positions throughout the lattice. Knowing $\beta$ at all points, one can describe the particle motion from one point to another as

$$
\left[\begin{array}{l}
x \\
x^{\prime}
\end{array}\right]_{s_{2}}=M\left(s_{1} \rightarrow s_{2}\right)\left[\begin{array}{l}
x \\
x^{\prime}
\end{array}\right]_{s_{1}}
$$

where

$$
M\left(s_{1} \rightarrow s_{2}\right)=\left[\begin{array}{cc}
\left(\frac{\beta_{2}}{\beta_{1}}\right)^{1 / 2}\left(\cos \Delta \psi+\alpha_{1} \sin \Delta \psi\right) & \left(\beta_{1} \beta_{2}\right)^{1 / 2} \sin \Delta \psi \\
-\frac{1+\alpha_{1} \alpha_{2}}{\left(\beta_{1} \beta_{2}\right)^{1 / 2)}} \sin \Delta \psi+\frac{\alpha_{1}-\alpha_{2}}{\left(\beta_{1} \beta_{2}\right)^{1 / 2}} \cos \Delta \psi & \left(\frac{\beta_{1}}{\beta_{2}}\right)^{1 / 2}\left(\cos \Delta \psi-\alpha_{2} \sin \Delta \psi\right) .
\end{array}\right]
$$

The phase advance $\Delta \psi$ in this matrix represents the phase advance from $s_{1}$ to $s_{2}$. Finally, this phase advance in a single pass system may be represented as,

$$
\Delta \psi\left(s_{1} \rightarrow s_{2}\right)=\int_{s_{1}}^{s_{2}} \frac{d s}{\beta(s)} .
$$

A more in depth derivation of the equations in this section can be found in [3].

\subsubsection{Quadrupoles and Thin Lenses}

Quadrupole magnets are used in the process of keeping a particle beam in a stable condition. A restoring force on a particle displaced from the design trajectory is required to keep this beam stable. One would like this restoring force to be as strong as possible. Using one of maxwell's eqauations, $\nabla \times \vec{H}=\vec{j}_{\text {free }}+\left(\frac{\partial \vec{D}}{\partial t}\right)$, and noting that the magnet is 
designed in such a way that there is an absence of free current $(J=0)$ and electric field $(\vec{E}=0)$, one is able to rewrite this equation as

$$
\nabla \times \vec{B}=0
$$

For equation 1.32 to hold, it is noted that all components of $\vec{B}$ must vanish simultaneously. If one examines the ŝ component,

$$
\nabla \times \vec{B}_{s}=\left(\frac{\partial B_{y}}{\partial x}-\frac{\partial B_{x}}{\partial y}\right) \hat{s}
$$

One may define two gradient terms,

$$
\begin{aligned}
B_{y}^{\prime} & =\frac{\partial B_{y}}{\partial x} \\
B_{x}^{\prime} & =\frac{\partial B_{x}}{\partial y}
\end{aligned}
$$

One desires a linearly proportional magnetic field, meaning that the farther a particle is away from it's ideal trajectory, the stronger the kick it will receive. This is shown in figure 1.3 which shows the gradient of a hypothetical magnet.

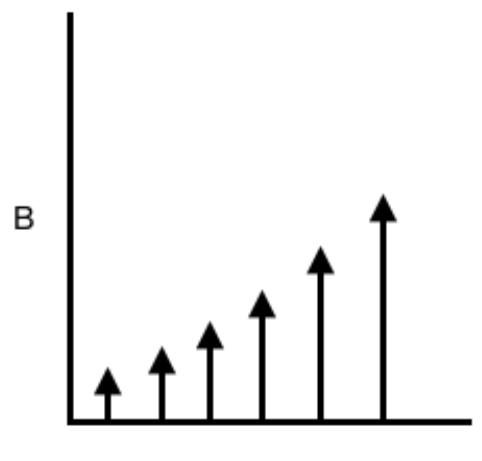

Position $\mathrm{x}$

Figure 1.3: Here one observes the theoretical gradient of a magnet. The more a particle strays from the ideal trajectory, the larger the kick it will recieve. 
Now, one must keep in mind that in order to satisfy this condition, both of these two gradients must add to equal zero, this means that one can only focus in one direction at a time. The solution to this problem is to alternate quadrupoles in such a way as to focus in one plane and then, at the next magnet, defocus in the same plane. This focusing/defocusing quadrupole technique is observed in figure 1.4. A more in depth derivation and analysis of this technique can be found in [3].

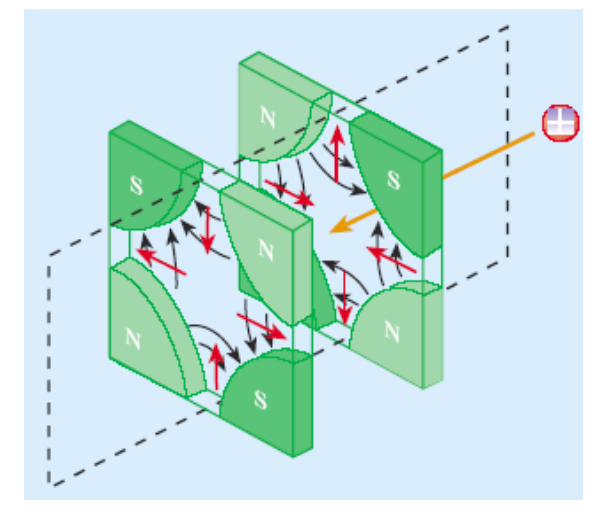

Figure 1.4: Here one two quadrupoles and a positively charged particle traveling through them. The first quadrupole the particle travels through is focusing in the horizontal plane, while the second is defocusing in the horizontal plane. The black lines in this figure represent the direction of magnetic field, while the red lines represent the direction of the force experienced by the particle traveling through the quad. The direction of the force received by the particle can be found by using the right hand rule.

Quadrupoles in particle accelerators can be thought of as optical thin lenses in cases where the focal length of the quadrupole is much larger than the length of the quadrupole. One can imagine a particle traversing a quadrupole at a distance $x$ from the magnet's axis of symmetry. This thin lens approximation means that the length of the magnet, $l$, is short enough so that the particles position $x$ is unaltered as it traverses the magnet. This means that the magnetic field experienced by the particle, $B_{y}=\left(\partial B_{y} / \partial x\right)$, is constant along the particle's trajectory, $x^{\prime}=d x / d s$. One can write the slope of a particle's transverse trajectory being altered as 


$$
\Delta x^{\prime}=-\frac{l}{\rho}=-l\left(\frac{e B_{y}}{p}\right)=-\left(\frac{e B^{\prime} l}{p}\right) x,
$$

where, as shown in figure 1.5, $\rho$ is the radius of curvature of the trajectory through the magnetic field and $B^{\prime}=\partial B_{y} / \partial x$ is the gradient of the quadrupole magnet. In equation 1.36 , it is noted that $1 / \rho=\frac{e B}{p}$ which follows from the Lorentz Force, equation 1.1.

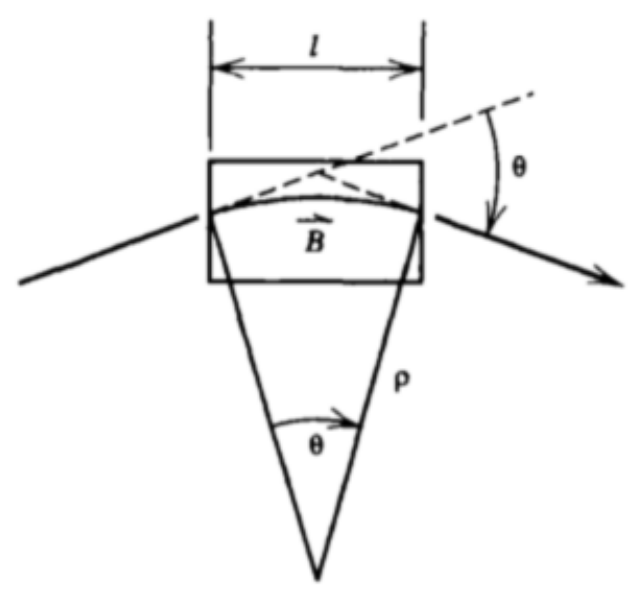

Figure 1.5: Here one observes the deflection of a particle by a thin lens magnetic element.

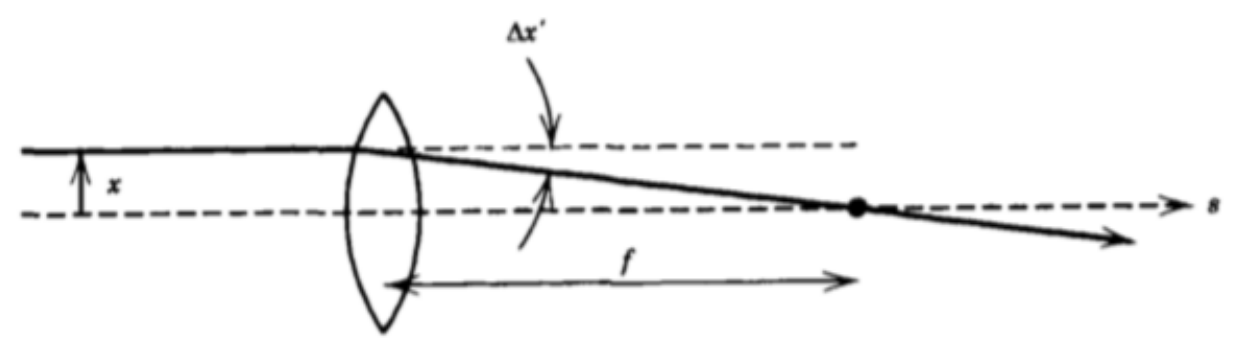

Figure 1.6: Here one observes a ray initially parallel to the optical axis. This ray is bent towards the optical axis, through the use of an optical thin lens, which causes the ray to pass through the focal point $f$.

If one now examines figure 1.6 , it is seen that the change in slope is $\Delta x^{\prime}=-x / f$, where $f$ is the focal length of the thin lens. Comparing this equation with equation 1.36, one obtains 


$$
\frac{1}{f}=\frac{e B^{\prime} l}{p} .
$$

Here one can observe the ratio $p / e$ which was earlier described as the magnetic rigidity, written as $(B \rho)$. It follows that equation 1.37 can be re-written as

$$
\frac{1}{f}=\frac{B^{\prime} l}{(B \rho)} .
$$

One may now wish to write a matrix that represents the passing of a particle through a "thin lens" quadrupole. To do this, equation 1.36 is written in matrix form

$$
\left[\begin{array}{l}
x \\
x^{\prime}
\end{array}\right]_{\text {out }}=\left[\begin{array}{cc}
1 & 0 \\
\pm \frac{1}{f} & 1
\end{array}\right]\left[\begin{array}{l}
x \\
x^{\prime}
\end{array}\right]_{\text {in }}
$$

In this matrix format, it is important to keep in mind if one is examining a focusing or defocusing quadrupole. If the quadrupole is focusing, the $1 / f$ term requires a negative sign out front and becomes $-1 / f$. If the quadrupole is defocusing, the $1 / \mathrm{f}$ term may remain as a positive value.

Similarly, one can also define a drift length $L$ between quads as the space between thin lenses. The transfer matrix for a drift space between lenses is,

$$
\left[\begin{array}{l}
x \\
x^{\prime}
\end{array}\right]_{\text {out }}=\left[\begin{array}{ll}
1 & L \\
0 & 1
\end{array}\right]\left[\begin{array}{l}
x \\
x^{\prime}
\end{array}\right]_{\text {in }} .
$$

\subsubsection{Dispersion}

Dispersion is a term that is used when studying particles that have momentum differing from that of the ideal particle. One can obtain dispersion from particles traveling 
through bending magnets that are set up for the ideal particle. Simply, high momentum particles will not bend quite as much as low momentum particles. One may determine the dispersion of a particle by examining the displacement off of the ideal orbit $x$ and it's momentum $p$ by the equation [3],

$$
D(p, s) \equiv x\left(\frac{\Delta p}{p_{0}}\right)^{-1} .
$$

In equation $1.40, x$ represents the a particle's horizontal displacement, $\Delta p$ represents the offset in a particle's momentum with respect to the momentum of a particle on the ideal trajectory, and $p_{0}$ represents the momentum of a particle on the ideal trajectory.

\subsubsection{Ellipses and Emittance}

In accelerator physics, it is often found useful to examine a particle distribution in terms of each particle's phase space coordinates. In most accelerator physics situations, the three planes can be thought of as decoupled. With decoupled planes, it is possible and useful to study a particle distribution's evolution through phase space independently in each plane. These phase space distributions are often approximated to be in the shape of an ellipse.

In order to understand emittance, one will find it useful to first examine the properties of an ellipse. While it may seem basic, understanding the math behind the area of an ellipse, proves to be a handy tool when trying to determine the emittance of a particle beam. Also in this section, another connection to the Courant-Snyder parameters used in Section 1.2.2 will be seen. A more detailed version of this sections derivations can be found at [4]. 


\subsubsection{Algebraic Formalism}

One may start with the general equation for an ellipse centered at the origin

$$
a x^{2}+b x y+c y^{2}=1
$$

From this general equation, one can solve for $y$ as a function of $x$ to arrive at

$$
y_{ \pm}=\frac{-b x \pm \sqrt{\left(b^{2}-4 a c\right) x^{2}+4 c}}{2 c} .
$$

It is known from the form of this equation, that it has two solutions. These two solutions correspond to the upper and lower portions of the ellipse, going from $x_{0}$ to $-x_{0}$. If one wishes to find the area of the ellipse, one simply needs to take the integral of the positive solution $y_{+}$and subtract the integration of the negative solution $y_{-}$to obtain

$$
A=2 \int_{-x_{0}}^{x_{0}} \frac{\sqrt{4 c-\left(4 a c-b^{2}\right) x^{2}}}{2 c} d x=\frac{4}{\sqrt{4 a c-b^{2}}} \int_{-1}^{1} \sqrt{1-u^{2}} d u
$$

Going through with the integration using $u=x / x_{0}$, one obtains an equation for the area of an ellipse

$$
A=\frac{2 \pi}{\sqrt{4 a c-b^{2}}}
$$

It is important to notice that the maximum/minimum value of the ellipse in the $x$ coordinate, or simillarly the $y$ coordinate, can be identified as

$$
\pm x_{0}= \pm(A / \pi) \sqrt{c}
$$


One will find it useful later in this chapter, to also derive the area of an ellipse using matrix notation.

\subsubsection{Matrix Formalism}

The first step in the matrix formalism is to obtain equation 1.41 using matrix multiplication. One can start by defining $\vec{X}$ to be

$$
\vec{X}=\left[\begin{array}{l}
x \\
y
\end{array}\right]
$$

and $\mathrm{A}$ as a general $2 \times 2$ matrix,

$$
A=\left[\begin{array}{ll}
a_{11} & a_{12} \\
a_{21} & a_{22}
\end{array}\right]
$$

If $\vec{X}^{T}$ is just the transpose of $\vec{X}$, one can multiply matrices together and set the product equal to a constant to obtain equation 1.41

$$
\vec{X}^{T} A \vec{X}=\left[\begin{array}{ll}
x & y
\end{array}\right]\left[\begin{array}{ll}
a_{11} & a_{12} \\
a_{21} & a_{22}
\end{array}\right]\left[\begin{array}{l}
x \\
y
\end{array}\right]=a_{11} x^{2}+\left(a_{12}+a_{21}\right) x y+a_{22} y^{2}=1
$$

Comparing the previous result for the area of an ellipse, equation 1.44 , one can find another equation for the area of an ellipse in terms of the new matrix formalism

$$
A=\frac{2 \pi}{\sqrt{4 a c-b^{2}}}=\frac{2 \pi}{\sqrt{4 a_{11} a_{22}-4 a_{12}^{2}}}=\frac{\pi}{\sqrt{\operatorname{det} A}} .
$$

Where $\operatorname{det} A$ is 


$$
\operatorname{det} A=\left(a_{11} * a_{22}\right)-\left(a_{12} * a_{21}\right)
$$

\subsubsection{Sigma Matrix}

One may now wish to find an ellipse that can be used to define the general shape and orientation of a particle beam. To begin, one can define a $\Sigma$ matrix by multiplying the two matrices $\vec{X}$ AND $\vec{X}^{T}$, and then taking the average of each element.

$$
\left\langle\vec{X} \vec{X}^{T}\right\rangle=\left\langle\left[\begin{array}{l}
x \\
y
\end{array}\right]\left[\begin{array}{ll}
x & y
\end{array}\right]\right\rangle=\left[\begin{array}{cc}
\left\langle x^{2}\right\rangle & \langle x y\rangle \\
\langle x y\rangle & \left\langle y^{2}\right\rangle
\end{array}\right]=\left[\begin{array}{cc}
\sigma_{11} & \sigma_{12} \\
\sigma_{21} & \sigma_{22}
\end{array}\right] \equiv \Sigma
$$

The inverse of $\Sigma$ is

$$
\Sigma^{-1}=\left[\begin{array}{cc}
\sigma_{22} & -\sigma_{12} \\
-\sigma_{12} & \sigma_{11}
\end{array}\right] \frac{1}{\operatorname{det} \Sigma} .
$$

If the inverse $\Sigma$ matrix is now used to create an ellipse, using the same method of creating an ellipse as the previous section, one obtains an equation of the form

$$
\sigma_{22} x^{2}-2 \sigma_{12} x y+\sigma_{11} y^{2}=\operatorname{det} \sum .
$$

It is important to remember that the $\Sigma^{-1}$ matrix was used to form this equation for an ellipse. Remember the equation for the inverse of a determinant

$$
\operatorname{det} \Sigma=\frac{1}{\operatorname{det} \Sigma^{-1}},
$$

Now referring to the result in equation 1.49 and substituting, one obtains 


$$
\sigma_{22} x^{2}-2 \sigma_{12} x y+\sigma_{11} y^{2}=(A / \pi)^{2}
$$

The foundation for finding an equation for the emittance of a particle beam has no been lain. In the next section, The techniques covered in the previous three sections will be used to arrive at a useful equation for finding emittance.

\subsubsection{Emittance}

Now that the tedious work of defining the area of an ellipse in different ways has been done, one is equipped to find an equation for emittance. One now refers to the transverse

coordinates $x$ and $x^{\prime}$, where $x$ is the distance from the ideal trajectory, and $x$ is the slope of that trajectory, $x^{\prime}=d x / d s$.

The reader is reminded that equation 1.30 shows that the maximum/minimum extent of the ellipse in either the $x$ or $y$ coordinate is proportional to the square root of the area of the ellipse. $\varepsilon$ is now introduced as the area of the phase space distribution one is studying.

Defining $x^{\prime}$ as the $y$ variable in the previous sections, one can now, once again, define new variables, called the Courant-Snyder parameters, to relate $\Sigma$ to the area of the ellipse.

$$
\begin{gathered}
\alpha \equiv-\sigma_{12} /(\epsilon / \pi)=-\left\langle x x^{\prime}\right\rangle /(\epsilon / \pi) \\
\beta \equiv \sigma_{11} /(\epsilon / \pi)=\left\langle x^{2}\right\rangle /(\epsilon / \pi) \\
\gamma \equiv \sigma_{22} /(\epsilon / \pi)=\left\langle x^{\prime 2}\right\rangle /(\epsilon / \pi)
\end{gathered}
$$

As stated previously, the Courant-Snyder parameters govern the orientation and shape of the ellipse. The emittance, or $\varepsilon$, is what governs the actual size of the ellipse. 
By following the same procedure to find the equation for an ellipse as before [4], it can be shown that

$$
\gamma x^{2}+2 \alpha x x^{\prime}+\beta x^{\prime 2}=\epsilon / \pi
$$

This ellipse defines what is often times called the "rms emittance" and can be written as

$$
\epsilon=\pi \sqrt{\left\langle x^{2}\right\rangle\left\langle x^{\prime 2}\right\rangle-\left\langle x x^{\prime}\right\rangle^{2}}
$$

One notes that the emittance is a preserved quantity through adiabatic invariance [5]. This means that while the shape of the ellipse changes in shape over time, the area within that ellipse will remain the same. While adiabatic invariance holds true for beam traversing a lattice with no obstacles, one must keep in mind that traversing some material will cause a beam's emittance to change. More on this topic will be covered in Section 1.2.7. 


\section{CHAPTER 2}

\section{BEAM INTERACTIONS WITH MATTER}

\subsection{Momentum Spread Reduction}

When a particle passes through some energy absorbing material, all three components of the particle's momentum are reduced. As a bunch of particles pass through some energy absorbing material, one can lower the bunch's average momentum in exchange for an increase in transverse emittance. The efficiency and heating effects of the absorbing depends on the atomic number $\mathrm{Z}$ of the material, the $\mathrm{dE} / \mathrm{dx}$ of a material, and the radiation length of the material.

The goal will be to use an energy absorbing material in the shape of a wedge to cool a muon beam. The wedge is to be placed in a position where there is beam dispersion, allowing high momentum particles to travel through the thickest part of the wedge, and vice versa. This technique will reduce the spread in the momentum of the bunch, allowing for more particles to be near the desired momentum and therefore able to be accepted into the storage ring.

Figure 2.1 represents a theoretical situation in which an experiment is originally set up to run for a number of years. This setup distribution is represented by the black line. The vertical red lines represent the momentum spread acceptance window, meaning only the particles within those vertical red lines can be used for the experiment. Now, say that the blue line represents the same setup distribution, but now the energy absorbing wedges are in place. One can observe the the blue line distribution has a much smaller spread 


\section{Particle Density}

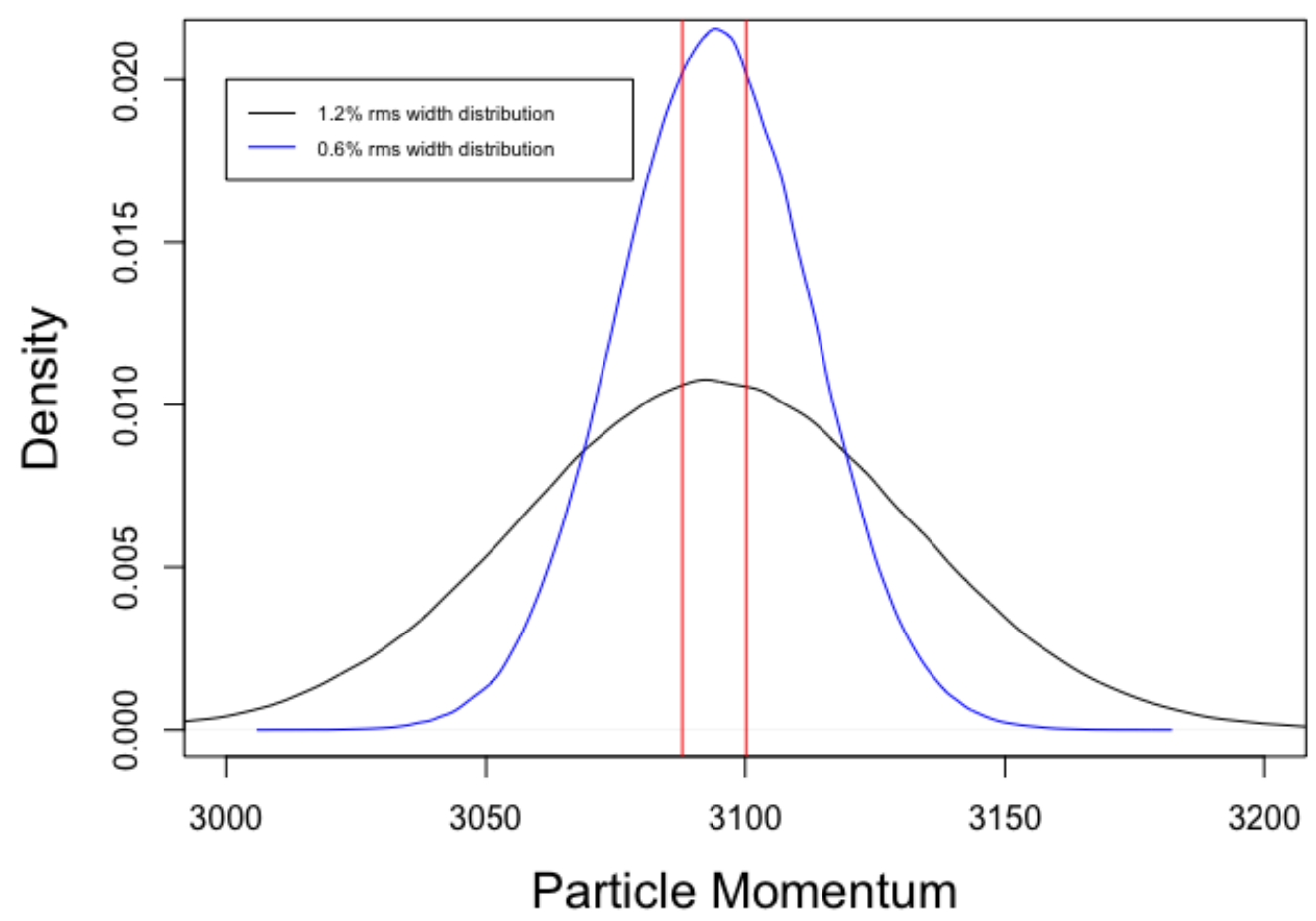

Figure 2.1: The black line represents a muon beam with a $1.2 \%$ rms width while the blue line represents a muon beam with a $0.6 \% \mathrm{rms}$ width. The vertical red lines represent the $0.2 \%$ acceptance range of the the storage ring in the $\mathrm{g}-2$ experiment.

in momentum when compared to the black line distribution. The blue line distribution is much more densely populated at its core and stores about twice as many muons in the storage ring as compared to the black line. If twice as many muons are stored within the acceptable momentum range, the experiment run time can potentially be cut in half. Using this technique, a research facility could cut experiment run time in half and save significant time and money.

Another aspect of the particle beam that one must keep in mind is the spin. Earlier, in chapter 1 , it is stated that the measurement of the magnetic dipole moment depends 
on the how particles of certain spin, or polarization, interact with a magnetic field. Given that this experiment heavily depends on the polarization of the particles, one must ensure that the polarization of the particles is not affected while traversing the energy absorbing wedge. In chapter 4 , the effects of the wedge on the polarization of the beam will be examined.

In order to gain a deeper understanding of how an energy absorbing wedge works, it will be found useful to examine the Bethe-Bloch Equation.

\subsubsection{Bethe-Bloch Equation}

In order to understand how an energy absorbing wedge will be useful, it is important to note the well-described Bethe-Bloch equation

$$
\left\langle-\frac{d E}{d x}\right\rangle=K z^{2} \frac{Z}{A} \frac{1}{\beta^{2}}\left[\frac{1}{2} \ln \frac{2 m_{e} c^{2} \beta^{2} \gamma^{2} W_{\max }}{I^{2}}-\beta^{2}-\frac{\delta(\beta \gamma)}{2}\right]
$$

This equation describes mean rate of energy loss, where $K$ is a constant, $Z$ is the atomic number of the absorber, $A$ is the atomic mass of the absorber, $W_{\max }$ is the maximum energy transfer to an electron in a single collision, $m_{e}$ is the mass of an electron, $\delta(\beta \gamma)$ is the density effect correction to ionization energy loss, and $I$ is the mean excitation energy. These coefficients are well known values that can be obtained from the "Particle Data Group" or the PDG for example. [6].

For particles traveling through a short length of material, one can say that the $d E / d x$ experienced remains constant. The goal is to influence the higher $\Delta p / p$ particles to travel through more material, causing a reduction in the spread of $\Delta p / p$. As a result of this reduction in momentum spread, one will ultimately store more muons for an experiment as in figure 2.1 . 


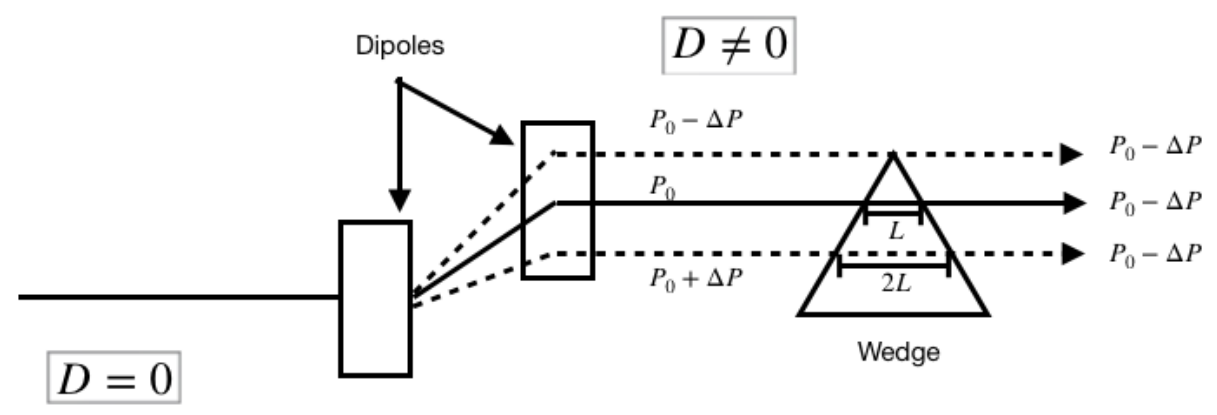

Figure 2.2: A particle beam obtains a non-zero dispersion value through the use of dipole bending magnets. A wedge of energy absorbing material is placed in the beams path.

Imagine three particles traveling along the same trajectory, but with three values of momenta: $P_{0}-\Delta P, P_{0}$, and $P_{0}+\Delta P$. By using two dipole magnets one can separate the trajectories, as indicated in figure 2.2. Next, a wedge-shaped object is placed in the path of the particles, such that its tip is on the $P_{0}-\Delta P$ trajectory. Finally, the angle of the wedge is adjusted so that the path length $\mathrm{L}$ for particle $P_{0}$ reduces its momentum by $\Delta P$. Likewise, the path length $2 \mathrm{~L}$ for particle $P_{0}+\Delta P$ will reduce its momentum by $2 \Delta P$. Thus, the momentum spread to has been reduced to zero.

In a more realistic scenario, a particle of momentum $P_{0}$ may traverse a different length of the wedge than the designed length $\mathrm{L}$ as described in figure 2.2 . This would cause particle $P_{0}$ to not lose exactly $\Delta P$ momentum. As a result, particle $P_{0}$ will not be at $P_{0}-\Delta P$ momentum upon exiting the wedge. This more realistic scenario of particles traversing more/less of the wedge than designed will result in a non-zero momentum spread after the wedge material. It is important to note that while the momentum spread after the wedge material will be non-zero, it will still be reduced when compared to the momentum spread before the wedge. In chapter 4 , the changes in momentum spread through a wedge of energy absorbing material will be examined.

While the beam is passing through some material, there will be two competing processes taking place. The first of these two processes is momentum cooling from ionization 
of the material. The second process taking place, which can cause a problematic increase in beam size, is scattering from Coulomb interactions. The objective, in order to reduce momentum spread, is to have the cooling processes outweigh the effects of the scattering processes. A more detailed look into the Bethe-Bloch Equation can be found at [7].

\subsection{Emittance Growth}

As mentioned in the previous section, the effects of the cooling processes a desired to outweigh the effects of the heating processes as the beam traverses the wedge. In this case, the heating processes arise primarily from Coulomb Scattering events.

\subsubsection{Coulomb Scattering}

As the beam traverses an energy absorbing material, it will experience many smallangle scatters. As these small deflections are randomly caused by Coulomb scattering, it is safe to assume that the displacements due to Coulomb scattering will produce a Gaussian distribution. A detailed analysis in [8] shows the rms width of the angles in the $\mathrm{x}$-plane, and similarly in the $\mathrm{y}$-plane, is given by

$$
\theta_{0}=\frac{13.6 M e V}{\beta c p} z \sqrt{\frac{x}{X_{0}}}\left[1+0.038 \ln \left(\frac{x z^{2}}{X_{0} \beta^{2}}\right)\right]
$$

In this equation, $p$ is the momentum, $\beta c$ is the velocity, and $z$ is the charge number of the incident particle. $x / \mathrm{X}_{0}$ is the defined as the thickness of the scattering medium in radiation lengths. 
If one considers the rms emittance given by equation 1.60 , it is noticed that it depends on both $x$ and $x^{\prime}$. Traversing a small length of energy absorbing material will not affect the $x$ parameter in a noticeable fashion. However, equation 2.2 shows that the rms angle of trajectory, $x^{\prime}$, for particles traversing a wedge will be affected in some way. One may write an equation for $x^{\prime}$ after some scattering of $\Delta \vartheta$ as

$$
x^{\prime}=x_{0}^{\prime}+\Delta \theta
$$

and

$$
\left\langle x^{\prime 2}\right\rangle=\left\langle\left(x_{0}^{\prime}+\Delta \theta\right)^{2}\right\rangle \text {. }
$$

If $\left\langle\Delta \vartheta^{2}\right\rangle^{1 / 2}$ is defined as the rms width of particle trajectory as defined in equation 2.2. It can be shown that an increase in $\left\langle\Delta \vartheta^{2}\right\rangle^{1 / 2}$ leads to an increase in emittance through equation 1.60 [10].

\subsubsection{Wedge Consideration}

It should be noted how that the creation of dispersion just before the wedges, as will be done in simulation, will allow for the Bethe-Bloch equation and Coulomb Scattering equations to act differently on particles traveling through different parts of the wedge. Calculating $\mathrm{dE} / \mathrm{dx}$ and $\mathrm{rms}$ scattering angles would become a tedious task when dealing with a wedge-like material. For this reason, one can leverage computer simulations to calculate energy loss and scattering angles based on the incoming particles trajectory, the material of the wedge, and the user defined geometrical properties of the wedge. 


\section{CHAPTER 3}

\section{THE FERMILAB MUON BEAM}

\subsection{Introduction}

At Fermilab, a series of beamlines have been designed and commissioned in order to deliver muon beams to the Fermilab Muon g-2 experiment. In this section, a brief description of the transport beam and delivery ring will be given. A more in depth analysis of the following can be found in [11].

\subsection{Beam Formation and Transport}

To create muons for the g- 2 experiment, a bunched beam of protons is taken from the recycler and directed through the M1 line to a pion production target. Once these pions of about $3.11 \mathrm{GeV} / \mathrm{c}$ have been created, they are directed through the M2 and M3 lines and into the Delivery Ring (DR) where the pions may decay into the required muons. The average pion has a lifetime of about 26.033ns. After the pions have decayed, the surviving muons are collected and brought through the M4 and M5 lines to the muon storage ring for experimentation.

The muon beam enters through a hole in the magnet of the storage ring, and then crosses into a near field free region, which delivers the beam to the edge of the storage region. The geometry of the storage ring does not allow for much error as it is quite constrained. A kick is required to put the muon beam onto a stable orbit. The strength 

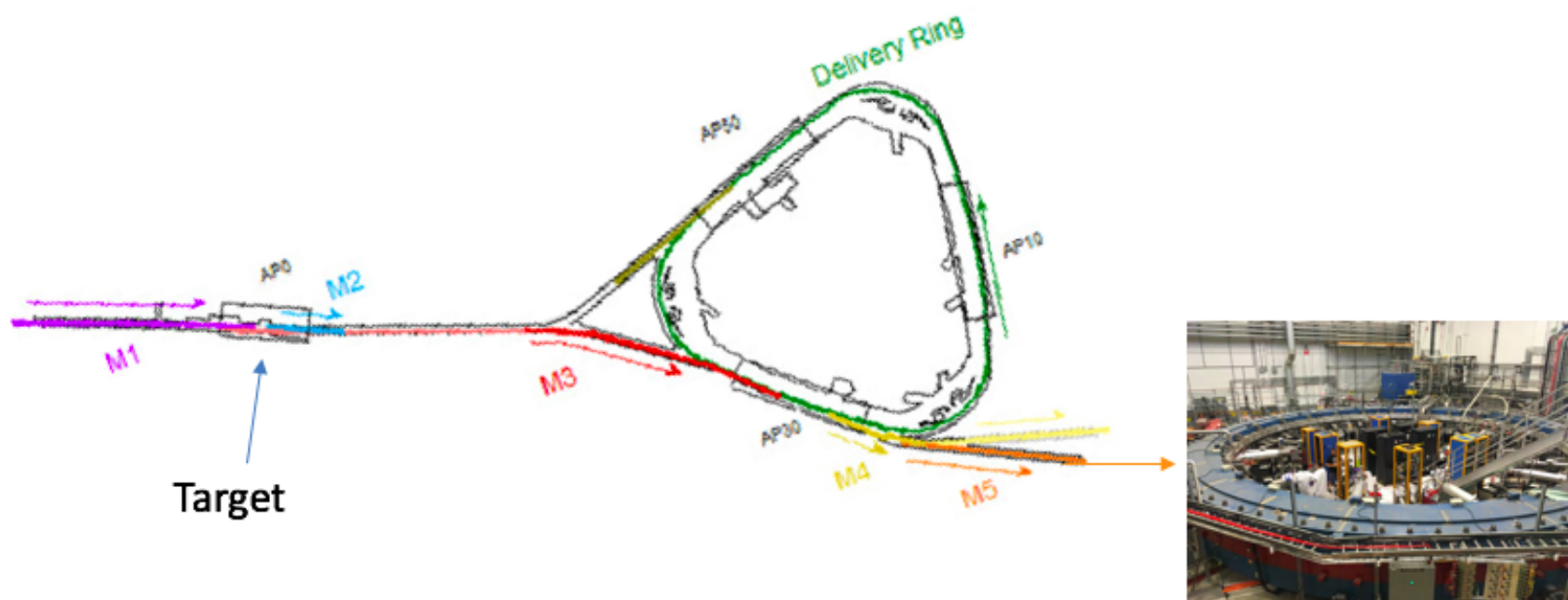

Storage Ring

Figure 3.1: Here one observes the series of beamlines necessary to deliver muons to the g-2 storage ring.

of this kick is determined in such a way as to put muons with the "magic momentum" of $3094 \mathrm{MeV} / \mathrm{c}$ onto orbit. The kick required to put these magic momentum muons onto a stable orbit is of the order of $10 \mathrm{mrad}$. The term "magic momentum" is defined from the spin precession equation defined later in this chapter.

The muon kicker requirements inside the storage ring are quite strict given the nature and space limitations of it. Given that the magnet is continuous inside the storage ring, the kicker is required to be inside of the precision magnetic field. It follows that the kicker cannot contain any magnetic elements since it has to be placed inside the magnetic field.

Once the muon beam is kicked onto a stable orbit within the storage ring, it is within a constant magnetic field. What one wishes to observe while these muons are circulating is how they interact with this strong constant magnetic field. The rate at which the muon spin turns relative to its momentum depends on the anomaly value of interest, $a_{\mu}$, and the average magnetic field experienced by the muon. If one is to obtain an accurate reading of $a_{\mu}$, then one must also have accurate measurements of the rate at which the muon 
polarization turns relative to it's momentum, $w_{\mathrm{a}}$, and the average magnetic field $\langle B\rangle$. Given that the muon beam is confined to a $9 \mathrm{~cm}$ diameter, it is clear to see why the average magnetic field experienced and momentum of the muons be well known.

Inside the storage ring, two frequencies are measured. The rate at which the muon polarization turns relative to the momentum is called $w_{\mathrm{a}}$, whereas the value of the magnetic field is normalized to the Larmor frequency of a free proton is called $w_{\mathrm{p}}$. One may define $w_{\mathrm{a}}$ as

$$
\vec{w}_{a}=\vec{w}_{S}-\vec{w}_{C}
$$

where S and C simply represent "spin" and "cyclotron". This spin precession is governed by the Thomas-BMT equation [20]:

$$
\begin{array}{r}
\vec{\omega}_{a}=-\frac{e}{m}\left[a \vec{B}-a\left(\frac{\gamma}{\gamma+1}\right)(\vec{\beta} \cdot \vec{B}) \vec{\beta}\right. \\
\left.-\left(a-\frac{1}{\gamma^{2}-1}\right) \frac{\vec{\beta} \times \vec{E}}{c}\right]
\end{array}
$$

In the absence of electric fields, one may represent $w_{\mathrm{S}}$ and $w_{\mathrm{C}}$ as

$$
\begin{gathered}
w_{S}=-g \frac{Q e}{2 m} B-(1-\gamma) \frac{Q e}{\gamma m} B ; \\
w_{C}=-\frac{Q e}{m \gamma} B .
\end{gathered}
$$

Using these definitions of $w_{\mathrm{S}}$ and $w_{\mathrm{C}}$,

$$
w_{a}=w_{S}-w_{C}=-\frac{g-2}{2} \frac{Q e}{m} B=-a \frac{Q e}{m} B
$$


Now in the presence of an electric field, the equation for $w_{\mathrm{a}}$ is modified to become

$$
\vec{w}_{a}=-\frac{Q e}{m}\left[a_{\mu} \vec{B}-\left(a_{\mu}-\left(\frac{m c}{p}\right)^{2}\right) \frac{\vec{B} \times \vec{E}}{c}\right] .
$$

If the experiment is operated with muons at the magic momentum of $3.094 \mathrm{GeV} / \mathrm{c}$, the electric field contribution cancels in the first order. This allows the experiment to use electric fields for focusing of the muon beam during storage in an ultra-uniform magnetic field. In order to determine $w_{\mathrm{p}}$, the magnetic field can be weighted by the muon distribution, as well as averaging over the running time weighed by the number of stored muons.

One now has what is needed to write an equation for the anomalous magnetic moment $a_{\mu}$

$$
a_{\mu}=\frac{w_{a} / w_{p}}{\lambda_{+}-w_{a} / w_{p}}=\frac{R}{\lambda_{+}-R},
$$

where $\lambda_{+}=\mu_{\mu}+/ \mu_{P}=3.183345137$ is the ratio of muon to proton magnetic moment, which has separately been measured to high precision. A more complete analysis of the g-2 experiment is shown in [11].

\subsection{The Use of Wedges}

As stated in a previous section, the objective of the two wedges placed in the M5 line is to reduce the momentum spread in order to obtain more muons in the core of the momentum distribution. Having more muons in the core of the momentum distribution allows for more muons to be accepted by downstream experiments, such as the muon $\mathrm{g}-2$ experiment. 


\subsubsection{Choice of Wedge Material}

In order to obtain the optimal results from the wedges, one requires a material that won't create large scattering, is dense, and has a high radiation length. The material is desired to be dense in order to reduce the amount of space needed to place the wedge in the beam pipe. The radiation length must be high in order to reduce the amount of multiple scattering. There are numerous materials that can be used that fit this description, such as, lithium hydride $(\mathrm{LiH})$, beryllium, and polyethylene $\left(\mathrm{C}_{2} \mathrm{H}_{4}\right)$.

As studied in [12], it is shown that using lithium hydride results in less multiple scattering and energy straggling than using polyethylene. However, due to the cost of producing LiH wedges as well as some concerns in handling and safety, polyethylene seems to be a better choice. Polyethylene is a material that performs nearly as good as $\mathrm{LiH}$, is readily available, easy to produce and shape, and relatively cheap. Beryllium produces similar results as the other two materials, but has significant handling issues.

One may question the durability of polyethylene as well as how much power is being transferred to the wedges by each bunch of muons. Each bunch of muons in the Fermilab Muon g-2 experiment contains approximately $10^{5}$ muons. Each muon in these bunches loses an average of about $15 \mathrm{MeV}$ of energy as a result of traversing the wedges. If one wishes to calculate the amount of power in watts lost by the muons as a result of traversing the wedges, one may use the equation $W=\frac{J * N}{\Delta t}$, where $W$ is watts, $J$ is the energy of the bunch's average muon in joules, $\mathrm{N}$ is the number of muons per bunch, and $\Delta t$ is the change in time. Using this equation, and knowing from simulations that the average particle takes about 8 ns to traverse both wedges, one arrives at a result of about 30 watts being dissipated into the wedges. To put this into perspective, a typical incandescent 
lightbulb uses about 60 watts of power. In other words, the wedges are not receiving a significantly high amount of energy from each passing muon bunch.

\subsubsection{Need For Dispersion}

Before sending the beam through the two wedges, it is necessary to create some dispersion. Creating dispersion before the wedges allows the wedges to work to their full effect. To create dispersion before the two wedges, a bending dipole magnet is required. The higher momentum muons will not be affected as much by the dipole, while the lower momentum muons will be affected more, causing dispersion. After creating beam dispersion, the beam is sent through the wedges. The wedges are placed in such a way so that the high momentum muons will travel through the thickest part of the wedge, while the lower momentum particles will travel through the thinner part of the wedge. Ideally, this will cause the momentum spread of the bunch to be reduced.

\subsubsection{How a Wedge Might Help}

In the muon g-2 experiment, about $10^{5}$ muons are delivered to the end of the M5 line per bunch, while only about $3-5 \%$ will be accepted into the storage ring [11]. The large percentage of muons that don't make it into the storage ring is mostly due to momentum spread $\Delta P / P$. Through the use of wedges, one can reduce the $(\Delta P / P)_{\mathrm{rms}}$, which will result in more muons being accepted and stored within the storage ring. 


\subsubsection{Ideal Parameters Before The Wedges}

As stated in the previous section, it is necessary to create dispersion in order for the wedges to be effective. Dispersion, however, is not the only beam parameter that one must study in order to maximize the effectiveness of the wedges. Courant-Snyder parameter values, for example, also play some role in effectiveness of the wedges. It is shown in chapter 4 that reducing the $\beta$ and $\alpha$ values in the $x$ and $y$ directions is useful.

\subsubsection{Recapturing The Beam}

Sending a particle beam through two plastic wedges alters the beam parameters quite a bit. In order for one to use these wedges and still get the beam to the storage ring, it is necessary to correct the beam parameters after the wedge. In order to recapture the beam, one is are able to modify the strengths of quads. However, one cannot just adjust quads to whatever values one sees fit, as there are limitations to the adjustments. Chapter 4 explores the quadrupole current limits as well as some quadrupoles being hooked up to the same power supply. When more than one quadrupole is hooked up to a given power supply, that means that altering the power supply for one quadrupole will also affect the others.

\subsection{Wedge Position}

Given that the wedges require beam dispersion in order to be utilized effectively, it becomes necessary to search the M5 line for a location with non-zero beam dispersion to 
place the wedges. By examining figure 3.4a, one notices that there is a location with nonzero dispersion at about $20 \mathrm{~m}$ on the horizontal axis. Figure $3.4 \mathrm{~b}$ represents a zoomed in version of figure 3.4a where the focus is now on the region of dispersion. Two vertical dotted lines have been placed in figure $3.4 \mathrm{~b}$ to show roughly where the wedges will be placed. The wedges are placed in these positions in the M5 line in order to utilize the dispersion that has been created.

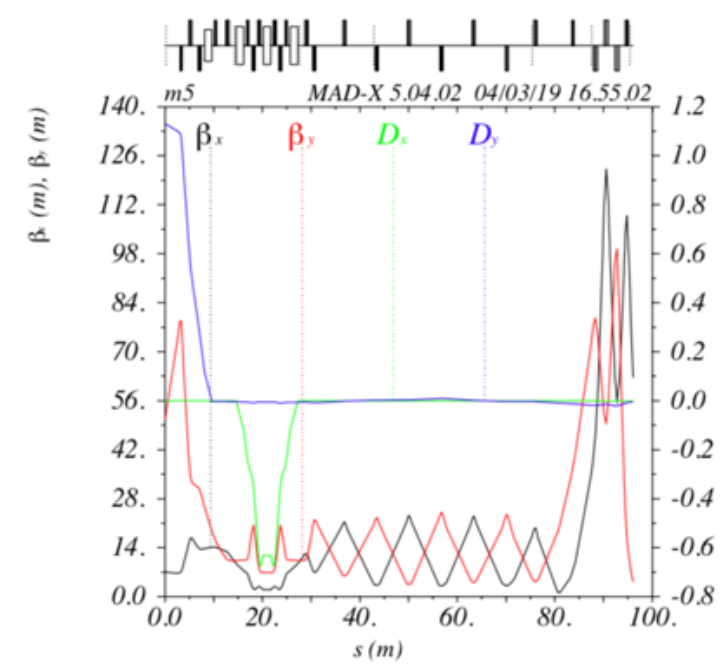

(a)

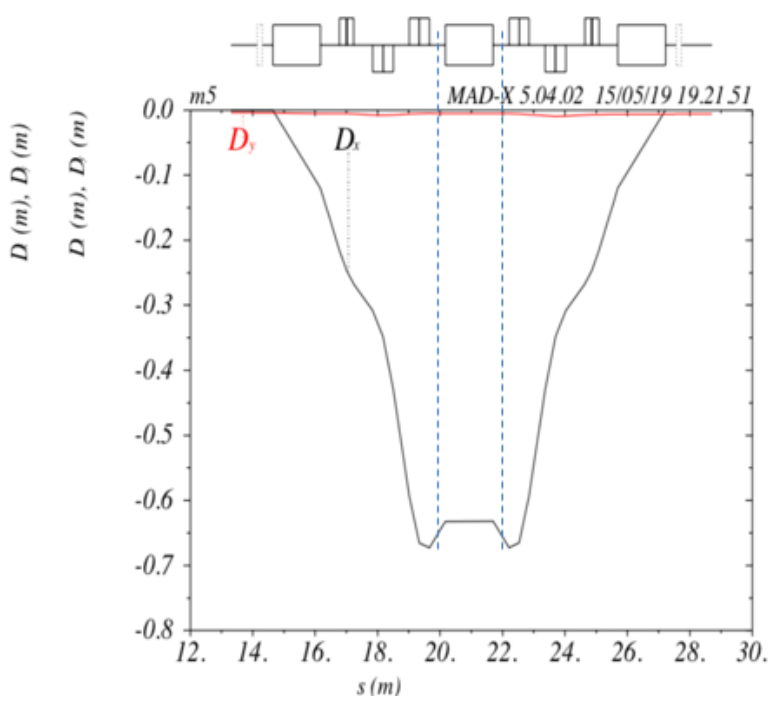

(b)

Figure 3.2: Figure (a) represents the $\beta$ and $D$ values in each transverse coordinate through the entire M5 line. Figure (b) represents a zoomed in view of the M5 line at a point where there is non-zero $D$ values in each transverse coordinate. Both of these figures are with the wedges removed.

The wedges are located between two quadrupoles and separated by a dipole as shown in figure 3.3 


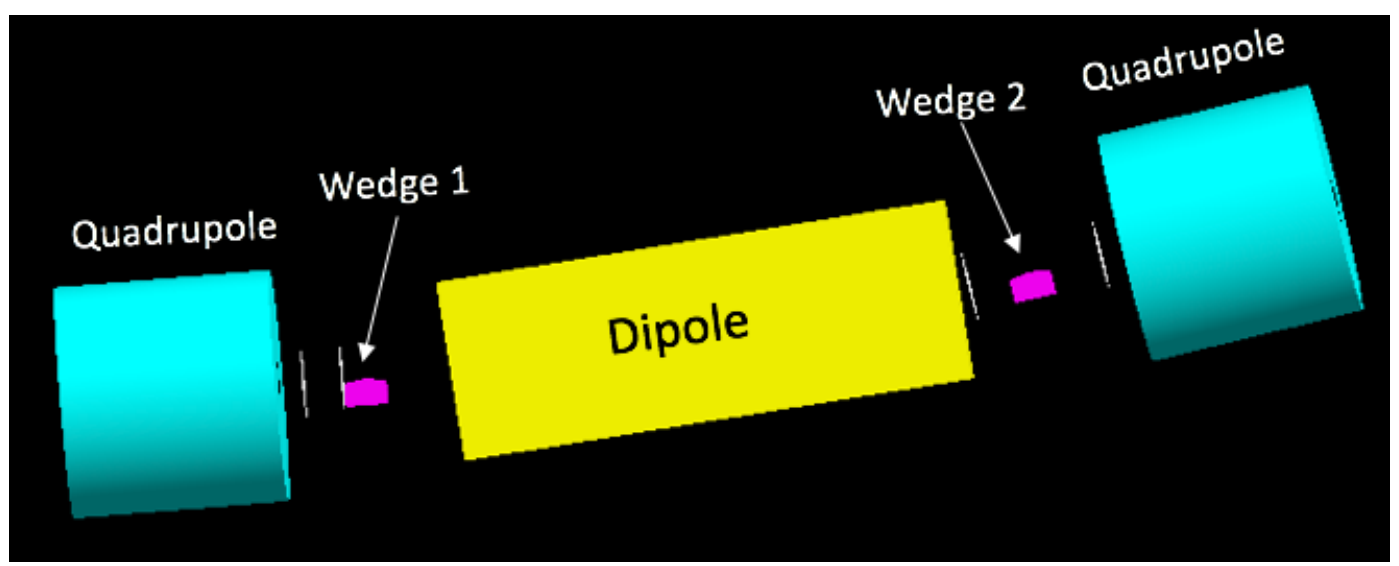

Figure 3.3: Here one observes the series of beamlines necessary to deliver muons to the g-2 storage ring.

\subsection{Wedge Specifications}

As stated previously, traversing polyethylene will lead to a reduced spread in momentum of a muon distribution. It is important to note that after a muon distribution traverses more than $250 \mathrm{~mm}$ of polyethylene, there is no additional increase in particles within the $0.2 \%$ momentum acceptance window [9]. The wedges to be used in the Fermilab Muon g-2 experiment are each $125 \mathrm{~mm}$ in length, $50 \mathrm{~mm}$ in height, and $80 \mathrm{~mm}$ in width as shown in figure 3.4 . The reason there are two wedges of $125 \mathrm{~mm}$ instead of one wedge of $250 \mathrm{~mm}$ is because there is not enough physical space to place a wedge of length $250 \mathrm{~mm}$ in the M5 line at a location of non-zero dispersion. A picture of one of the wedges to be used for the Fermilab Muon g-2 experiment can be seen in figure 3.5. 


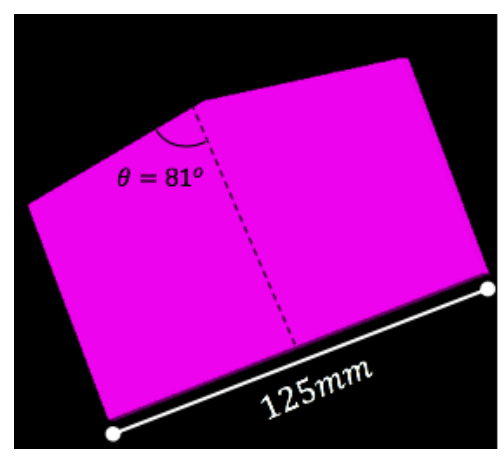

(a)

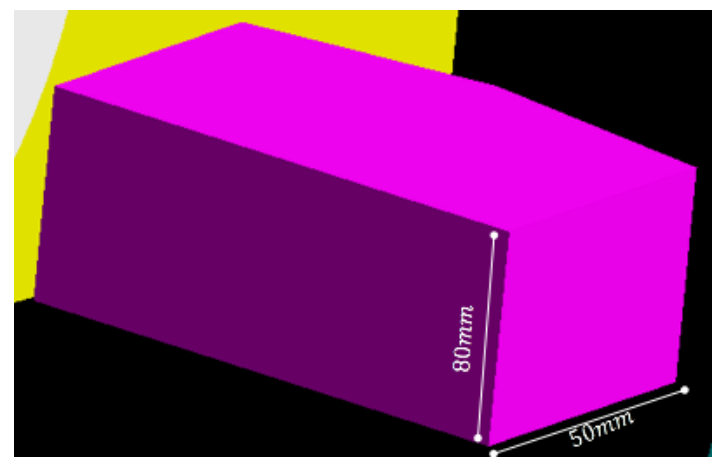

(b)

Figure 3.4: Figure (a) shows the top view of each wedge while figure (b) shows the height and width of each wedge.

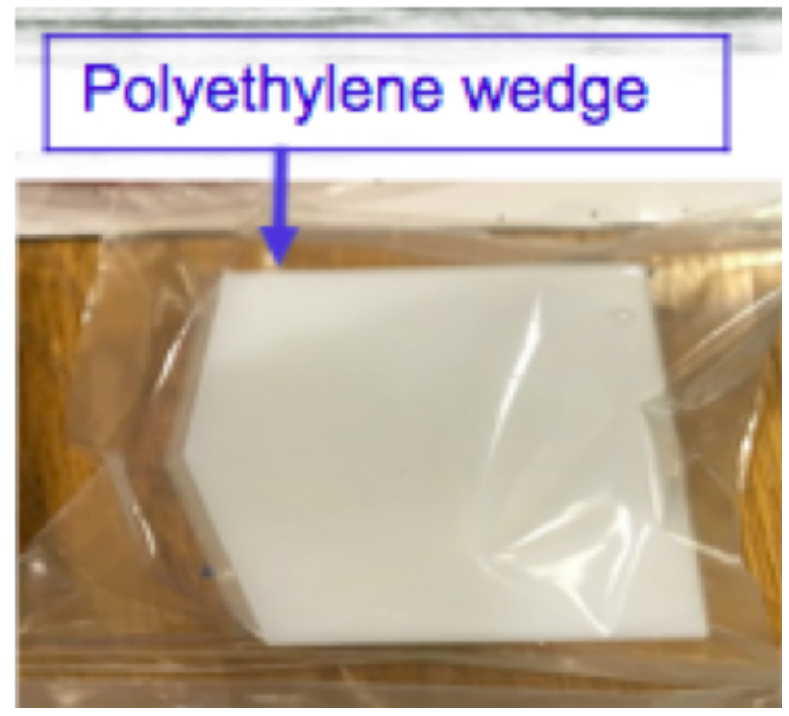

Figure 3.5: Here, one observes a picture of one of the actual polyethylene wedges to be used in the Fermilab Muon g-2 experiment. 


\section{CHAPTER 4}

\section{SIMULATIONS AND RESULTS}

\subsection{G4beamline}

G4beamline is a particle tracking software that can be easily used to simulate sending beam through a lattice [13]. G4beamline tracks particles through a lattice by using equations stated in chapter 1, such as the Lorentz Force equation, in small iterations. Creating a lattice in G4beamline follows the same style of coding used in other lattice building programs. One begins by defining elements, such as quads, dipoles, detectors, and drift lengths. It is also important to note, for the purpose of this paper, that G4beamline allows one the freedom to define a wedge in which particles may travel through. Along with defining these elements, one can define the geometry, material, and strengths of the elements as well. G4beamline also allows one to define the beam that one wishes to send through your lattice. The user has the freedom to decide the initial beam parameters, type of particle, and the number of particles to simulate. The above is a brief description of G4beamline. For a more extensive description of its capabilities, see [13].

Once the user has all of the elements for use defined, one can start placing the elements. In G4beamline, each element placed in the lattice begins where the previous element has ended. While the particle beam that has been defined is traveling through the lattice, G4beamline keeps track of key parameters such as the position and momentum, along with many other parameters, in each of the three degrees of freedom. The user has the ability to alter the step size for physics calculations. It should be noted that 
while having a smaller step size will increase the accuracy of a simulation, it will also increase the run time and computing power required. Once the end of the defined lattice is reached, the user will find it useful to have the tracked parameters printed out to a user friendly text file. This can be done with the simple use of a single command.

As mentioned before, the freedom to define a wedge and track particles traveling through it makes G4beamline an extremely valuable resource in the analysis.

\subsection{MADX}

MADX provides an easy way to track useful parameters of a beam such as the CourantSnyder parameters, Dispersion, and many others [14]. MADX tracks these parameters through the matrix approaches discussed in chapter 1. One feature of MADX that one will find very useful, is how simple it is to create intuitive visualizations. Much like G4beamline, MADX also provides a way to produce a simple output text file of the beam parameters through each element as well as the position of each element.

One crucial difference between MADX and G4beamline, is the ability of G4beamline to define a wedge and track particles traveling through it. In addition, G4beamline also offers the capability of tracking particles through realistic apertures of magnetic elements. Another key difference is the ability of MADX to match user parameter restrictions at certain points of the lattice. It is for this reason, that using both MADX and g4beamline proves to be very useful. While MADX and G4beamline are used independently, it can be of benefit to use them in unison. For example, in Section 4.8 a case will be observed in which G4beamline is used to track particles traveling through the energy absorbing wedges. G4beamline will supply the user with the beam parameters immediately after the wedges. The user can take those beam parameters from immediately after 
the wedges and use them to track the courant snider parameters through the rest of the lattice using MADX.

\subsection{Objective of Simulation}

As previously stated, the goal of the simulations is to provide reliable data to show that the use of two wedges in the M5 line produces a higher number of muons in a smaller momentum range. In order to reach the goal, one will want to reduce the standard deviation in momentum of the particle bunch. If one can reduce the standard deviation in momentum of the bunch, then one has now acquired more particles at the target momentum value. For example, in order for the muons to be accepted into the muon g-2 storage ring, they need to be within $0.2 \%$ of the magic momentum. Having more particles at the target momentum value would then lead to an increase in stored muons. In the following sections, $\mathrm{R}$ was leveraged to code the mathematical necessities [15] and the NERSC computing cluster was used to run large g4beamline simulations [18]. Each NERSC computing simulated 70,000 particles traversing the M5 lattice. Each simulation took 4 computing nodes about 5 minutes to complete.

\subsection{Ideal Beam Parameters}

One may start the analysis by looking to the parameters of the beam. Ideally, large dispersion just before the beam traverses through the wedges is desired. This dispersion allows for the separation of high momentum particles and low momentum particles in the bunch. Recall that in order for the wedge to be effective, it is required that the high momentum particles travel through the thickest part of the wedge, while the low mo- 
mentum particles travel through the thinnest. Figure 2.2 outlines the creation and use of dispersion clearly.

Next, one may wish to study how the emittance changes in the transverse coordinates as the particles traverse through the wedges. It is important to keep in mind that while the standard deviation in momentum may be decreasing, the emittance in each of the transverse coordinates may be greatly increasing. If the emittance in the transverse coordinates become too large, one will lose particles to the walls of the beam pipe.

\subsection{Custom Beam Parameters Simulation}

In order to study how to reduce the transverse emittance growth after traversing the wedges, one needs to be capable of creating beam distribution input files with custom defined parameters. This is an important step in the analysis since g4beamline requires the user to input a text file that describes the initial conditions of the beam.

The objective in this section is to study how the wedges affect momentum spread and transverse emittance immediately after the second wedge. To do this, one can take the parameters from a virtual detector that has been strategically placed, in g4beamline, after the second wedge. In the following sections it will be observed how to modify a distributions parameters before the wedges. Being able to modify a distributions parameters before the wedges allows one to observe how different beam parameters affect emittance growth after the wedges. The goal is to find which modified beam parameters provide the lowest amount of emittance growth after the wedges. 


\subsubsection{Analysis}

To begin the analysis, it is required to build the M5 line lattice in g4beamline. I've been supplied with a lattice file and a particle input distribution by Diktys Stratakis, through private communication, which uses the lattice specifications outlined in the fermilab g-2 technical design report [11]. This base lattice file and input distribution will serve as the starting point for the analysis to come. Another analysis done using these provided data can be found at [16].

Using this base input distribution and the M5 lattice file, one can simulate a beam traveling through the M5 lattice. The distribution's Courant-Snyder parameters after the second wedge can give one useful insight as to how the wedges are performing. The goal is to leverage g4beamline to study the Courant-Snyder parameters and momentum distribution at this position and to obtain evidence that the wedges are an asset to the experiment.

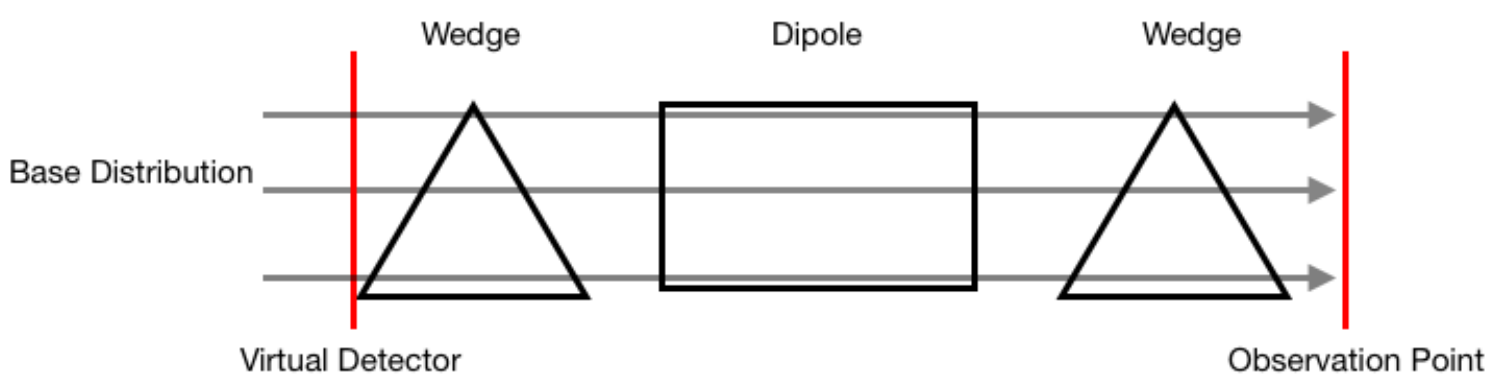

Figure 4.1: Here one observes the base distribution passing through the first virtual detector and both wedges on it's way to the observation point. The observation point is itself another virtual detector in G4beamline.

Since G4beamline doesn't give the Courant-Snyder parameters directly, it becomes necessary to do some calculations to obtain them. The parameters of interest that G4beamline does supply, are the position and momentum in each of the three degrees of freedom. 
With only these parameters, one is able to arrive at values for the $D, \beta, \alpha, \gamma$, and $\varepsilon$. To do this, one may use the equations [19]:

$$
\begin{gathered}
D_{x}=\frac{\langle x \delta\rangle}{\left\langle\delta^{2}\right\rangle} \\
D_{x}^{\prime}=\frac{\left\langle x^{\prime} \delta\right\rangle}{\left\langle\delta^{2}\right\rangle} \\
\epsilon \beta=\left\langle x^{2}\right\rangle-\frac{\langle x \delta\rangle^{2}}{\left\langle\delta^{2}\right\rangle} \\
\epsilon \gamma=-\left\langle x x^{\prime}\right\rangle+\frac{\left\langle x^{\prime} \delta\right\rangle^{2}}{\left\langle\delta^{2}\right\rangle} \\
\epsilon \alpha=\left\langle x^{\prime 2}\right\rangle-\frac{\left\langle x^{\prime} \delta\right\rangle^{2}}{\left\langle\delta^{2}\right\rangle} \\
\epsilon=\sqrt{(\epsilon \beta)(\epsilon \gamma)-(\epsilon \alpha)^{2}}
\end{gathered}
$$

Where

$$
\delta=\frac{\Delta P}{P} .
$$

We're now capable of obtaining the Courant-Snyder parameters for the base input distribution at any point where a virtual detector has been placed in G4beamline. Given the ability to obtain the Courant-Snyder parameters throughout the lattice, one is now capable of modifying the base distribution to have user defined Courant-Snyder parameters. In modifying the base distribution, one will find it useful to keep some parameters of the base distribution the same. For example, the dispersion, $D$, before the wedges is already at an ideal value. 


\subsubsection{Base Distribution}

In order modify a distribution's parameters, one must first have a base distribution to build off of. A data set of beam parameters just upstream of the first wedge has been provided by Fermilab. The data set provided can be seen in use in [16]. The next step is to run this distribution through the created M5 lattice in G4beamline as shown in figure 4.1. Once the run is complete, one can examine how the beam parameters change as it traverses the lattice. For this simulations interests, one wishes to modify the base distributions parameters just before the first wedge. To do this, one may look to a virtual detector that has been placed just before the first wedge in G4beamline. One can modify the base distributions beam parameters at this point in the lattice. Once the base distributions parameters have been modified, the modified distribution is sent through the wedges to the observation point for further study as shown in figure 4.2 .

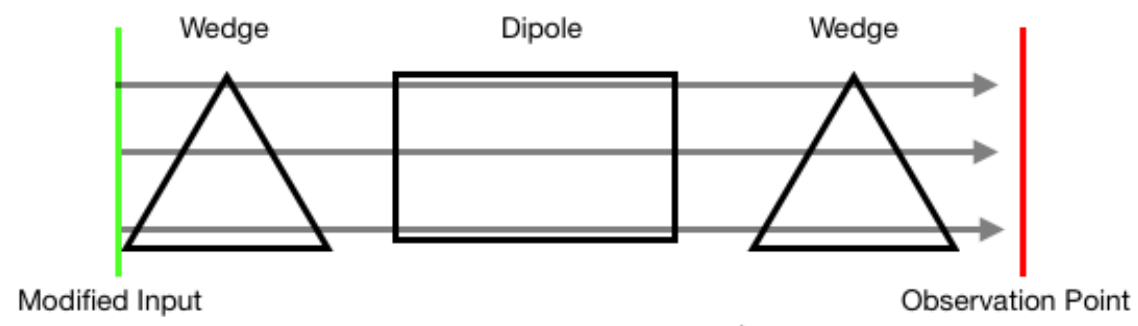

Figure 4.2: Here one observes the generated input distribution traversing through both wedges and to the observation point.

\subsubsection{Modifying Values}

As stated previously, to study how one can obtain the best performance out of the wedges, one wishes to modify the base distributions parameters just before the wedges. 
Modifying the base distribution with custom parameters allows one the freedom to study many different scenarios. For example, if one modifies the base distribution and simulates a run that results in less emittance growth and a smaller $\Delta P / P$ than the base distribution, perhaps the strengths of quadrupoles upstream can be altered to obtain the modified distribution's parameters.

One will find it useful to keep in mind that there are some parameters of the beam that one may wish to keep the same as one modifies the base distribution. The parameters which will be changed to obtain a modified distribution are the $\beta$ and $\alpha$ values. To do this, some equations to find $\beta$ and $\alpha$ some distance downstream are required

$$
\begin{gathered}
\beta_{2}=\beta_{1}-2 \alpha_{1} d+\gamma_{1} d^{2} \\
\alpha_{2}=\alpha_{1}-\gamma_{1} d
\end{gathered}
$$

where

$$
\gamma=\frac{1-\alpha^{2}}{\beta} .
$$

and $d$ is the distance between $\beta_{1}$ and $\beta_{2}$ or $\alpha_{1}$ and $\alpha_{2}$.

To modify the base distribution, it is required to solve these equations for $\beta_{1}$ and $\alpha_{1}$. The assumption is made that $\alpha_{2}$ is focused down to a value of zero. By assuming $\alpha_{2}$ is zero some distance downstream, one obtains

$$
\begin{gathered}
\beta_{1}=\beta^{*}+\frac{d^{2}}{\beta^{*}} ; \\
\alpha_{1}=\frac{d}{\beta^{*}}
\end{gathered}
$$


where $\beta_{1}$ and $\alpha_{1}$ are the values before the first wedge, while $\beta^{*}$ is the $\beta$ function at a focusing point some distance through a drift space of length $d$ away. In this simulation three different values of $d$ will be used, each of which represents the distance from the virtual detector before the first wedge, to some focal point downstream. The three focal points that will be used will be the midpoints of the first wedge, the dipole between the wedges, and the second wedge as shown in figure 4.3 .

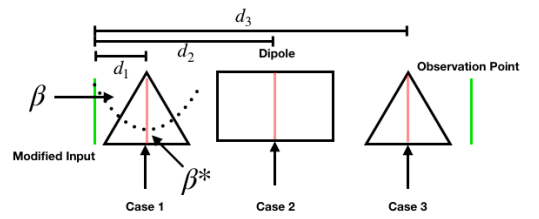

(a)

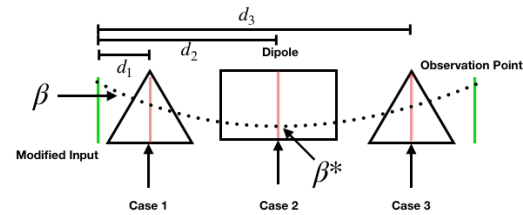

(b)

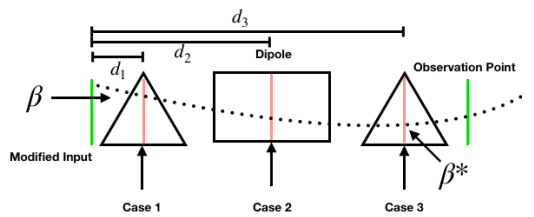

(c)

Figure 4.3: Figure (a) has $\alpha$ focused to zero at the first wedge, while (b) is focused at the dipole between the wedges and (c) is focused at the second wedge.

The target values for $\beta^{*}$ at each focal point for this simulation are 1 through 7 meters in each transverse coordinate. Running this simulation through this range of $\beta$ 's in each transverse coordinate allows one to create two matrices displaying emittance, one in the $x$ direction and one in the $y$ direction. Two matrices of emittance for each value of $d$ will be created. These created matrices will be used to create plots of emittance in the "Results" section.

\subsubsection{Modifying the Base Distribution}

In order to modify $\beta_{1}$ and $\alpha_{1}$ in the base distribution as described above, one needs to modify the base distribution's particle positions and momenta. By first examining a case where there is no dispersion, one can describe a particles transverse betatron motion, $x_{\beta}$, in terms of the Courant-Snyder parameters and emittance [19]. 


$$
\begin{gathered}
\left\langle x_{\beta}^{2}\right\rangle=\epsilon \beta \\
\left\langle x_{\beta}^{\prime 2}\right\rangle=\epsilon \gamma \\
\left\langle x_{\beta} x_{\beta}^{\prime}\right\rangle=-\epsilon \alpha
\end{gathered}
$$

Now, imagine that some dispersion is introduced, $D$, as is done just before the distribution is sent through the wedges. Introducing this dispersion allows for the particles to be dispersed by the value $D \delta$, where $D$ is simply the dispersion function and $\delta$ is the momentum deviation, $\Delta \mathrm{P} / \mathrm{P}$ from the ideal trajectory, of an individual particle. The value for $\Delta \mathrm{P} / \mathrm{P}$ that is used is the same $\Delta P / P$ that was acquired from the base distribution. This allows one to describe the position and trajectory of a particle with respect to the ideal position and trajectory as

$$
\begin{aligned}
& x=x_{\beta}+D \delta ; \\
& x^{\prime}=x_{\beta}^{\prime}+D^{\prime} \delta .
\end{aligned}
$$

In this analysis, the values of $D$ and $D^{\prime}$ were chosen to remain at the same values as the base distribution. One will also find it useful in the modification of the base distribution to define a particles momentum in the transverse coordinate $x$ as

$$
P_{x}=x^{\prime} P_{z}
$$

To determine the $x_{\beta}$ values, a random gaussian distribution with a $\sigma$ value of $\sigma=$ $\left(\varepsilon_{\mathrm{x}} \beta_{\mathrm{x}}\right)^{1 / 2}$ will be used, where $\varepsilon_{\mathrm{x}}$ is the emittance from the base distribution and $\beta_{\mathrm{x}}$ is the target $\beta$ value.

Determining the values for $x_{\beta}^{\prime}$ is also necessary. To do this, one may use the equation 


$$
x_{\beta}^{\prime}=\frac{P_{a d j}-A_{x} x_{b}}{B_{x}},
$$

where $\mathrm{P}_{\text {adj }}$ is obtained by creating another random gaussian distribution with the same $\sigma$ as found before, and $A_{x}$ is an $\alpha$ value of one's choosing.

The same treatment can be applied for the transverse coordinate $y$. Now that new values for $\mathrm{x}, \mathrm{x}, \mathrm{y}, \mathrm{y}^{\prime}$ and $P$ have been created, we're able to modify the base distribution's text file. Now that the base distribution has been modified, all that remains is to run G4beamline again. As stated previously, the custom distribution is desired to be inserted just before the wedges. In order to do this, one can modify the G4beamline starting position of the lattice file.

As before, one may take the parameters that $\mathrm{g} 4$ beamline supplies, and obtain values for $D, \beta, \alpha, \gamma$, and $\varepsilon$ immediately after the wedges.

\subsubsection{Results}

From the analysis, some useful plots can be put together for each of the three cases of distance $d$.

From the plots in figure 4.4, one observes how the emittance after the second wedge changes as the focused $\beta^{*}$ in each transverse coordinate is altered. It is quite evident in each of the three cases, that $\varepsilon_{\mathrm{x}}$ depends mostly on $\beta^{*}$ while $\varepsilon_{\mathrm{y}}$ depends mostly on $\beta_{\mathrm{y}}^{*}$. It appears that both $\varepsilon_{\mathrm{x}}$ and $\varepsilon_{\mathrm{y}}$ scale linearly with $\beta_{\mathrm{x}}^{*}$ and $\beta_{\mathrm{y}}^{*}$ respectively. Upon further

examination of figures $4.4 \mathrm{~b}, 4.4 \mathrm{~d}$, and $4.4 \mathrm{f}$, one notices that a $\beta_{\mathrm{x}}^{*}$ value of $1 \mathrm{~m}$ in each of these three cases results in a high $\varepsilon_{\mathrm{x}}$ after the second wedge. This is contradictory to the linear relationship observed in the rest of the figures. In other words, one would expect 


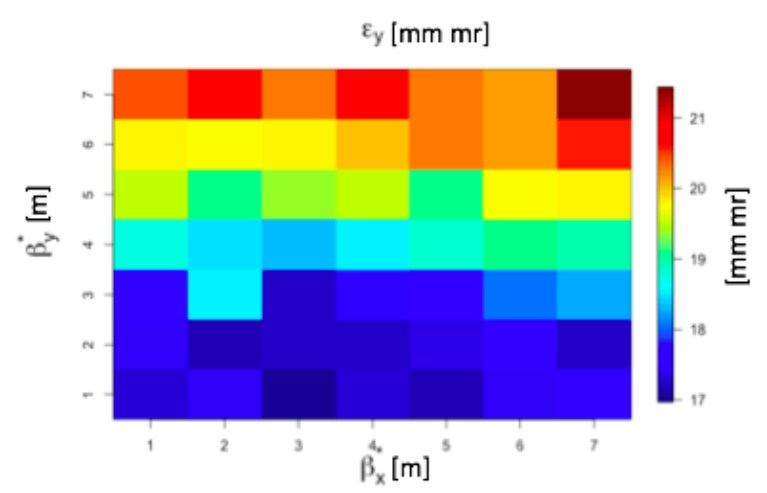

(a) Case $1 \varepsilon_{y}$

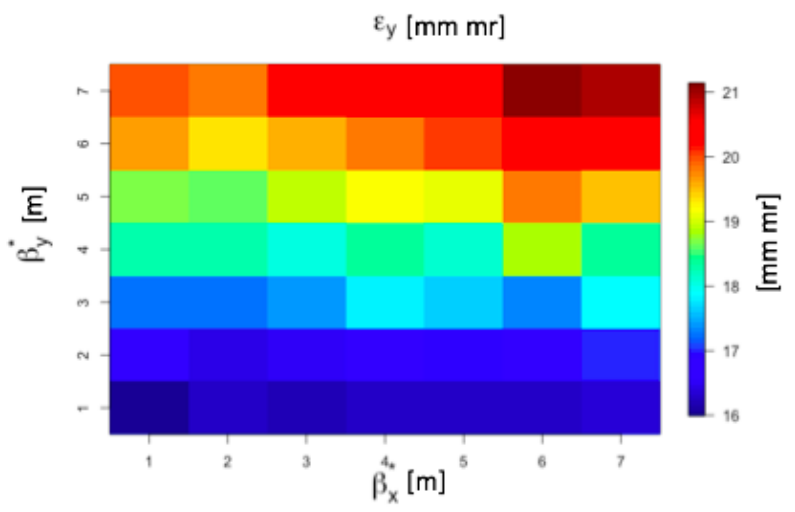

(c) Case $2 \varepsilon_{y}$

$\varepsilon_{\mathrm{y}}[\mathrm{mm} \mathrm{mr}]$

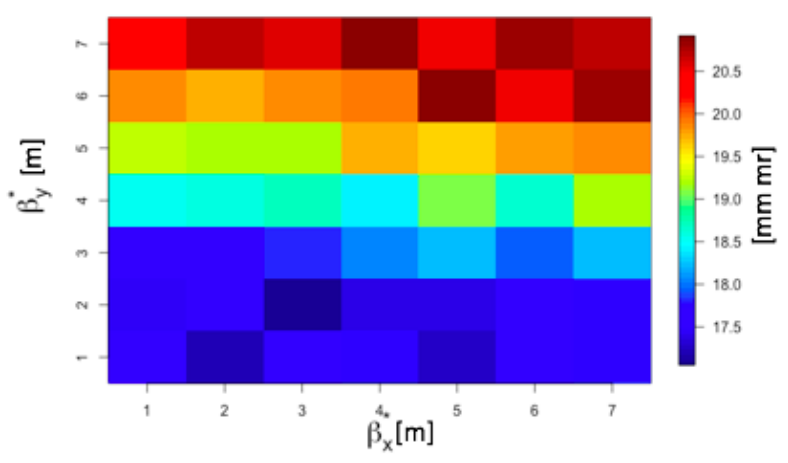

(e) Case $3 \varepsilon_{y}$

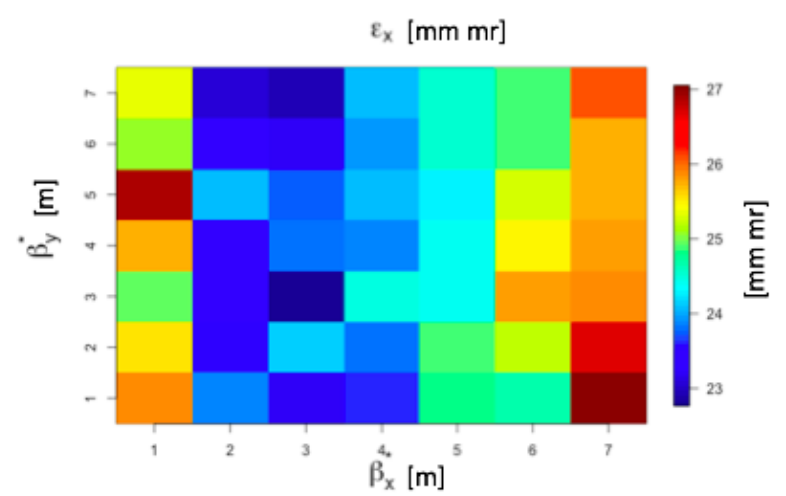

(b) Case $1 \varepsilon_{\mathrm{X}}$

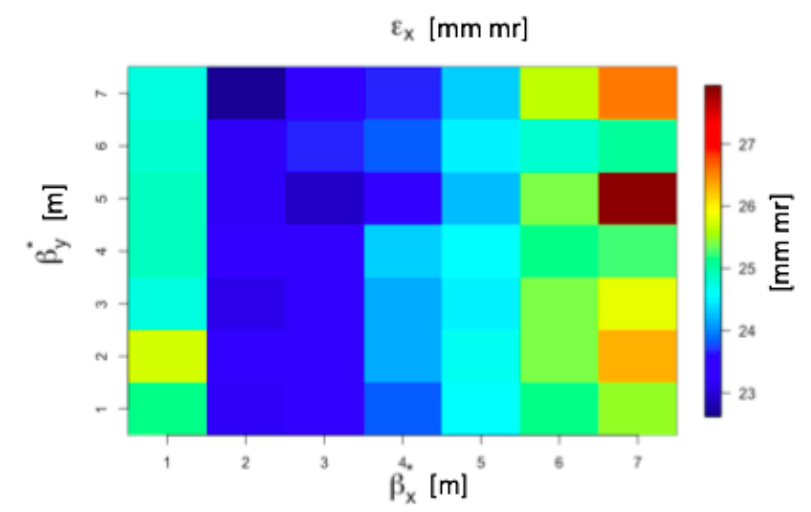

(d) Case $2 \varepsilon_{\mathrm{X}}$

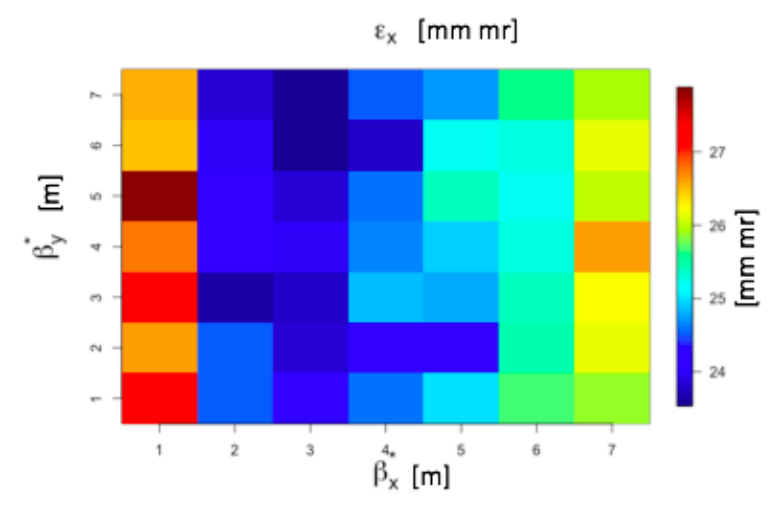

(f) Case $3 \varepsilon_{\mathrm{x}}$

Figure 4.4: The captions "case $(1,2,3)$ " refer to the three different distances of $d$ at which $\beta^{*}$ values are focused. Cases 1 and 3 refer to a focal point inside the first and second wedges respectively. Case 2 refers to a focal point inside of the dipole in-between the two wedges. The vertical axis in the images represents the changing $\beta_{y}$ value. The horizontal axis represents the changing of $\beta_{\mathrm{x}}$, while the colored tiles represent $\varepsilon_{\mathrm{y}}$ and $\varepsilon_{\mathrm{x}}$ values respectively. 
that a $\beta_{x}^{*}$ value of $1 \mathrm{~m}$ would result in the lowest $\varepsilon_{\mathrm{x}}$ value after the second wedge. To get a better idea of what could be causing this, one will find it useful to examine figure 4.5 .

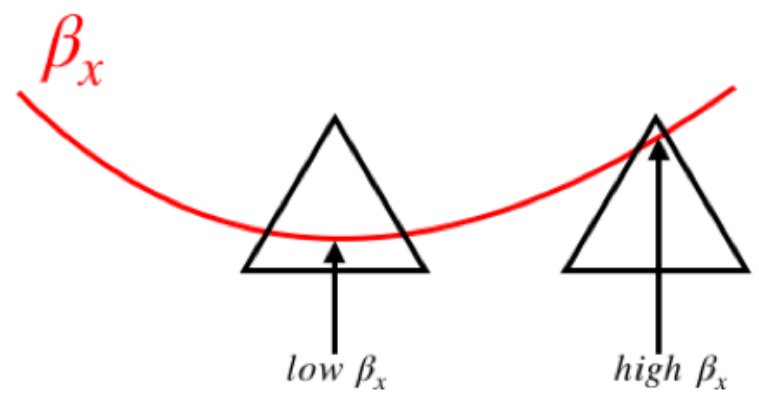

Figure 4.5: In this figure, one observes the effect of having a low $\beta^{*}$ value of $1 \mathrm{~m}$ at one of the wedges.

In figure 4.5, one observes that having a low $\beta^{*}{ }_{x}$ value of $1 \mathrm{~m}$ at one of the wedges results in a high $\beta_{x}$ value at the other wedge. The reader is reminded that a high $\beta_{x}$ at either of the wedges will lead to a high $\varepsilon_{\mathrm{x}}$ after the second wedge. One may conclude that a result of having a $\beta^{*}$ value of $1 \mathrm{~m}$ at one of the wedges, the $\varepsilon_{\mathrm{x}}$ value after the second wedge will be high.

It is noted that it is the $d$ value of case 2 in figure 4.4 that provides the lowest emittance growth after the second wedge. In case 2 , if $\beta_{x}^{*}$ and $\beta_{y}^{*}$ are each set to 2 , about a $8 \%$ decrease in $\varepsilon_{\mathrm{x}}$ growth and a $30 \%$ decrease in $\varepsilon_{\mathrm{y}}$ growth is observed when compared with a run with the base distributions parameters. The $\beta_{x, y}^{*}$ values of the base distribution are 3 and 7.3 respectively. A table of the $\varepsilon_{x, y}$ growth reductions in each of the three cases when compared to the base distributions $\varepsilon_{x, y}$ growth can be found in figure 4.6 .

While these plots are very useful, it is important to keep in mind that reducing the standard deviation in momentum is of higher priority. A useful treatment of the data would be to examine by how much one has reduced the standard deviation in momentum. Since case 2 was the most effective case, simulations at the $d$ value of case 2 are now of greatest interest. To observe the improvement, one can calculate the percent improve- 


\begin{tabular}{l|ccc} 
& \multicolumn{1}{c}{} & & \\
& Emittance Growth Reduction [\%] & & $\epsilon_{y}$ \\
\hline Case 1 - Optimized & $\epsilon_{x}$ & 22.9 \\
\hline Case 2 - Optimized & 7.46 & 30.3 \\
\hline Case 3 - Optimized & 8.13 & 22.3
\end{tabular}

Figure 4.6: In this figure, one can observe the optimized percent reduction in $\varepsilon_{x, y}$ growth for each of the three cases when compared to the base distributions $\varepsilon_{\mathrm{x}, \mathrm{y}}$ growth.

ment between the standard deviation in momentum, $P_{\mathrm{STD}}$, in the G4beamline run with wedges and a $d$ value of case 2 , and the run without wedges.

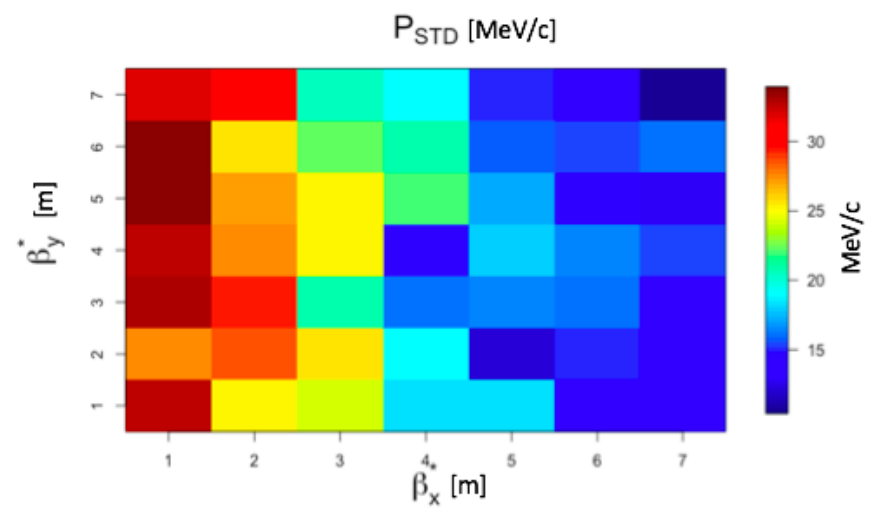

Figure 4.7: This figure represents the standard deviation in momentum of the beam bunch. The data in this plot are taken immediately after the second wedge in case 2 .

One observes in figure 4.7 that while changing $\beta_{y}^{*}$ does indeed alter the $P_{\mathrm{STD}}$, it is $\beta_{x}^{*}$ that has the greatest impact. This makes sense given that the wedge has been created in such a way as to utilize dispersion in the $x$ direction.

It would be ideal to use the created distributions with the lowest $\beta^{*}$ values in each transverse coordinate for the g- 2 experiment. In reality, one can only focus the $\beta^{*}$ values down to about $2.5 \mathrm{~m}$ for each direction. Given that this is a more realistic, yet still fairly low $\beta^{*}$ value, one could do a further study into how to achieve such parameters by altering quadrupole strengths upstream of the wedges. 


\subsection{Wedge Position Simulation}

In this section, the effects of the wedges positions at the end of the M5 line will be studied. Specifically, the amount of muons at the end of the M5 line are within $0.2 \%$ momentum acceptance window will be studied. Studying the momentum distribution at the end of the M5 line will give a better understanding as to how much of an improvement can be gained through altering the wedges positions.

For this sections analysis, operational quadrupole settings and g-2 beam distributions have been provided by Fermilab, this lattice is called “operational lattice 030519" and can be seen in use in [16]. This provided distribution is the result of an end-to-end simulation starting from the g-2 production target. The current quadrupole strengths and current M5 beam input file are not to be confused with the files representing the TDR quad strengths and M5 input file provided for Section 4.5. Using both of these files, one can simulate beam traversing the M5 line at the g-2 experiments current working conditions. An example of an analysis done with these data can be found at [17].

\subsubsection{Analysis}

For this analysis, G4beamline will once again be leveraged. As in Section 4.5, a virtual detector will be used to extract the beam parameters of interest at a given point in the lattice. The virtual detector, in this case, is placed at the end of the M5 line. The beam parameter of highest interest is the momentum of each particle, where:

$$
P=\sqrt{P_{x}^{2}+P_{y}^{2}+P_{z}^{2}}
$$


How the emittance changes at the end of M5, in each transverse coordinate, with the altering of the wedges positions will also be examined.

Unlike Section 4.5, one does not need to modify a base distribution for this analysis. Modifying a distribution to custom parameters was a useful tool used to study theoretical beam parameters, whereas now it is desired to study the operational g-2 experiment parameters.

A feature of G4beamline that will be found most useful here, is the ability to displace the wedges in the lattice through user input. To begin the wedge simulation process, a case where the first wedge is given the freedom to move $20 \mathrm{~mm}$ into or out of the beam path as shown in figure 4.8 will be examined. Displacing the wedge into or out of the beam causes the tip of the wedge to be at different positions in the beam path. If the wedge is in it's "unaltered" position, that means that it's position hasn't been displaced at all. This unaltered wedge position is the position in which the tip of the wedge is presumed to be at the center of the incoming particle bunch. One can observe the effect that displacing the wedge has on the amount of particles within the $0.2 \%$ momentum acceptance window at the end of M5. If it is found that displacing the wedge results in more particles around the target momentum, perhaps this suggests that the centroid of the particle beam is entering the wedge in a fashion differing from the ideal case in figure 2.2 .

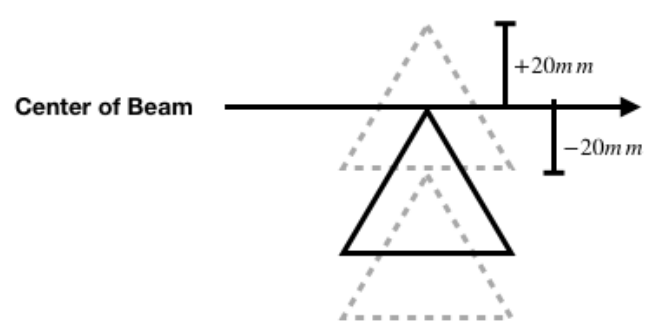

Figure 4.8: Moving the wedge into or out of the beam by $20 \mathrm{~mm}$ is represented by the dashed triangle while the solid triangle represents the original position of the wedge. 
The next wedge simulation is the opposite of the first. The first wedge will be removed from the lattice and the second wedge will be re-inserted. Once again, the wedge will be given the freedom to move $20 \mathrm{~mm}$ into or out of the beam path. As mentioned previously, an increase in particles around the target momentum as a result of displacing the wedge could mean the centroid of the particle beam is not entering the wedge as previously thought. It will be shown in the results section that removing either of the wedges more than $10 \mathrm{~mm}$ from the beam path has no effect on the number of muons within the momentum acceptance window at the end of M5.

The last simulation to perform, regarding wedge displacement, is to place both the wedges into the lattice and give them both the freedom to move $10 \mathrm{~mm}$ out of the beam path to $20 \mathrm{~mm}$ into the beam path. This simulation will pinpoint the position each wedge needs to be placed at in order to get the maximum number of muons within the momentum acceptance window. Each of these three cases will be examined in the next section.

\subsubsection{Results}

Using G4beamline's ability to displace the wedges, one can now produce some plots to tell which of these displacements will provide more muons within the momentum acceptance window. First, the first two cases where only one wedge is placed into the lattice will be examined.

In figure 4.9 one observes that displacing the second wedge into the beam path by about $6 \mathrm{~mm}$ results in the maximum amount of muons within the momentum acceptance range at the end of M5. The increase in muons is about $40 \%$ when compared to a run no wedge and $10 \%$ when compared to a run with the wedge set in an unaltered position

of $0 \mathrm{~mm}$. Similarly one sees an increase in particles within the momentum acceptance 
Particles at End of M5 (scaled)

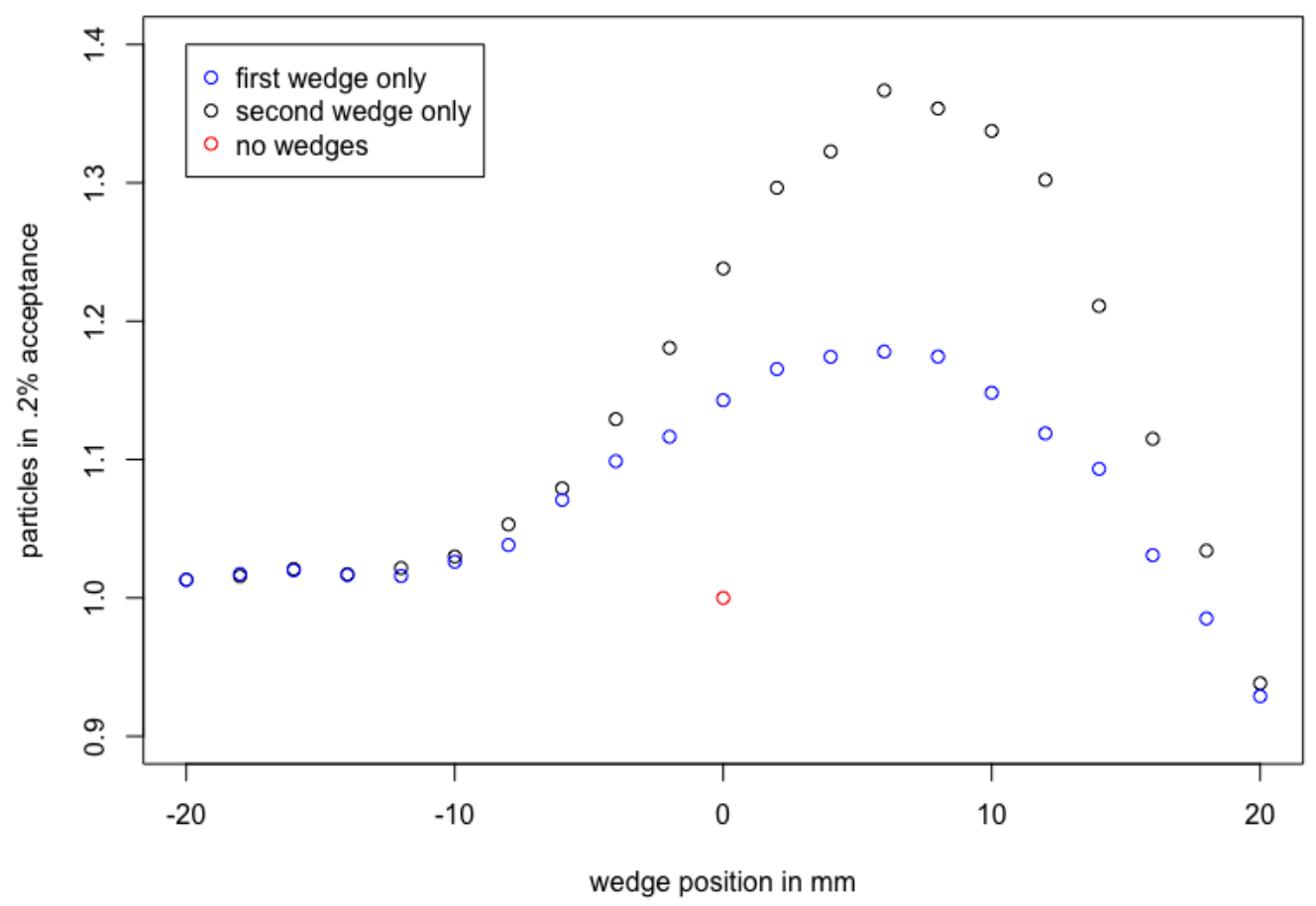

Figure 4.9: In this figure one sees how moving one wedge, with the other removed, affects the amount of particles within the $0.2 \%$ momentum acceptance window at the end of the M5 line.

range when the first wedge is moved $6 \mathrm{~mm}$ into the beam path. The increase in muons with only the first wedge placed in the lattice is about a $18 \%$ and $3 \%$ when compared to a run without a wedge and a run with the wedge in it's unaltered position respectively. Since inserting the wedges about $6 \mathrm{~mm}$ into the beam provides more particles within the momentum acceptance window than it's original $0 \mathrm{~mm}$ displacement, this is evidence that the centroid of the beam is actually $+6 \mathrm{~mm}$ from the design trajectory as well.

An important takeaway from figure 4.9 , is that the second wedge is performing much more effectively than the first wedge. In Section 4.6.2.1, some reasons as to why this is happening will be examined. 
Now let's examine the case in which both wedges are placed into the lattice and allowed them to move into or out of the beam path.

\section{Particles at end of M5 in Momentum Acceptance Window}

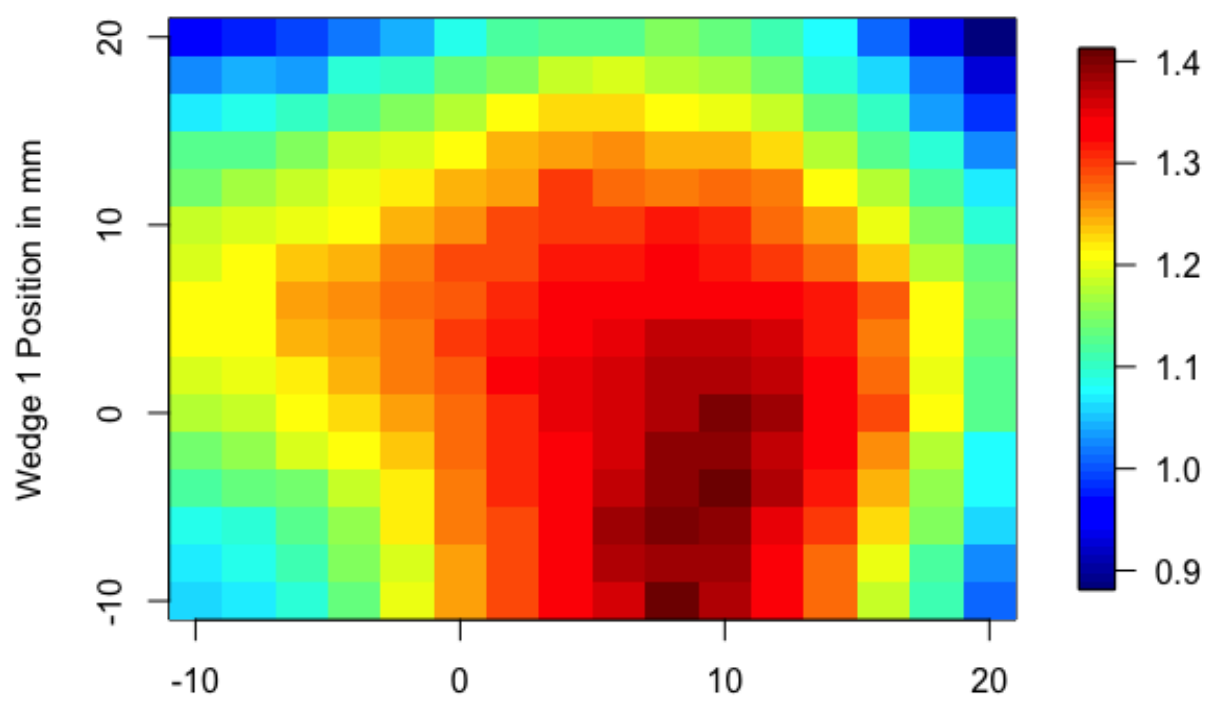

Wedge 2 Position in $\mathrm{mm}$

Figure 4.10: In this figure one observes a scenario in which both wedges are inserted into the lattice and are granted the freedom to move from $-10 \mathrm{~mm}$ out of the beam path to $+20 \mathrm{~mm}$ into the beam path. Here the $\mathrm{X}$ axis represents Wedge 2 and the $\mathrm{Y}$ axis represents Wedge 1 . This figure displays the amount of particles within the $0.2 \%$ momentum acceptance window at the end of the M5 line scaled to a run with no wedges in place.

In figure 4.10 , one observes how altering each wedge's position affects the amount of particles within the momentum acceptance window at the end of the M5 line. One can see that as in the first 2 cases, the second wedge seems to be the dominant, in terms of effectiveness, wedge. One can see this by looking to the horizontal and vertical axes of figure 4.10. It is observed that along the horizontal axis, the amount of particles within the momentum acceptance window changes much more rapidly than the vertical axis. In fact, it is observed that the maximum amount of particles are within the momentum 
acceptance window when the first wedge is almost completely removed and the second wedge is inserted about $8 \mathrm{~mm}$.

Next, lets examine how the emittance at the end of M5 has changed due altering both of the wedges positions. Before the wedges, the emittance values of the distribution are $11.7 \mathrm{~mm} \mathrm{mr}$ in the $x$ direction and $11.75 \mathrm{~mm} \mathrm{mr}$ in the $y$ direction. It is seen in figure 4.11 that the further the wedges are inserted into the beamline, the higher the emittance will be at the end of the M5 line. In a case where the wedges are in an unaltered position, the emittance values at the end of M5 are $15.5 \mathrm{~mm} \mathrm{mr}$ in the $x$ direction and $13.5 \mathrm{~mm}$ $\mathrm{mr}$ in the $y$ direciton. If one examines the emittance values at the end of M5 while both wedges are in optimal position as seen in figure 4.10 , when the first wedge is at $-10 \mathrm{~mm}$ and the second wedge is at $8 \mathrm{~mm}$, one observes a $\varepsilon_{\mathrm{x}}$ value of $15.7 \mathrm{~mm} \mathrm{mr}$ and a $\varepsilon_{\mathrm{y}}$ value of $13.7 \mathrm{~mm} \mathrm{mr}$. This means that even though the wedges positions have been altered to increase the amount of particles within the momentum acceptance window by about $10 \%$ when compared to when the wedges are in their unaltered positions, the emittance values in the $x$ and $y$ directions only grow by about $1 \%$ at the end of M5 when compared to the wedges being in their unaltered position. An increase in the emittance in both transverse coordinates of only $1 \%$ means that, downstream of the wedges, the beam was successfully recaptured. More on recapturing the beam will be discussed in Section 4.8.

From all of these scenarios one can conclude that with the current experimental beam parameters, the second wedge is more effective than the first wedge. Using the second wedge alone could yield up to about $40 \%$ more muons around the target momentum than without using any wedges. One also sees that if both wedges are used, one could obtain once again about $40 \%$ more muons around the target momentum than without using wedges. 


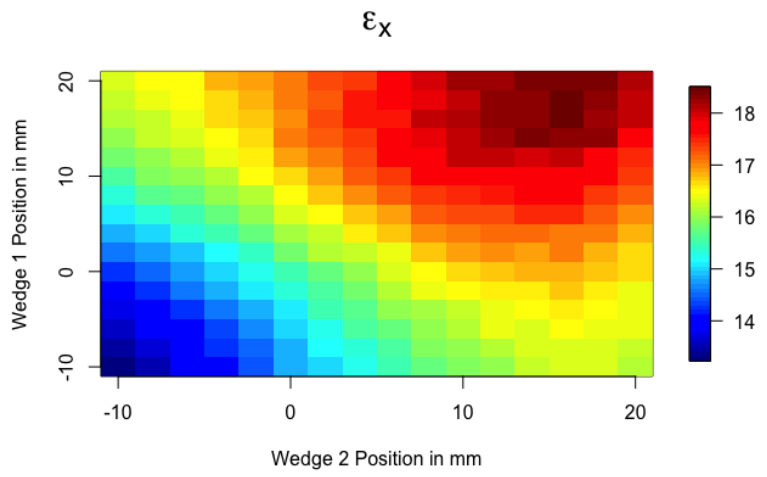

(a) $\varepsilon_{\mathrm{x}}$ at the end of M5

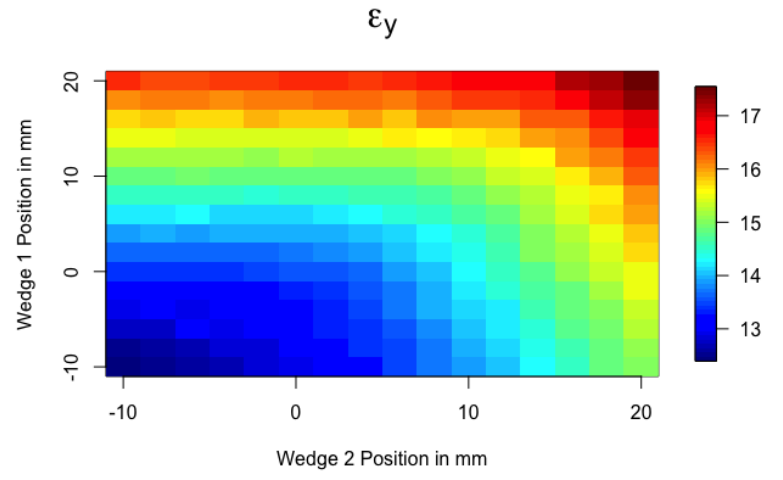

(b) $\varepsilon_{y}$ at the end of M5

Figure 4.11: Both (a) and (b) represent the emittance, $\varepsilon$, at the end of the M5 line the position of wedge 1 and wedge 2 are altered.

\subsubsection{Wedge Effectiveness}

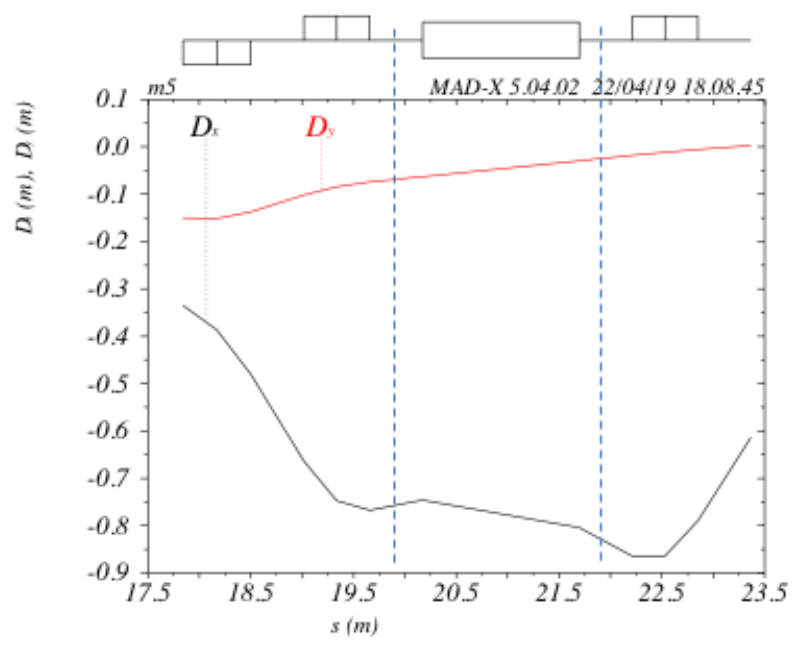

(a) $D_{\mathrm{x}, \mathrm{y}}$

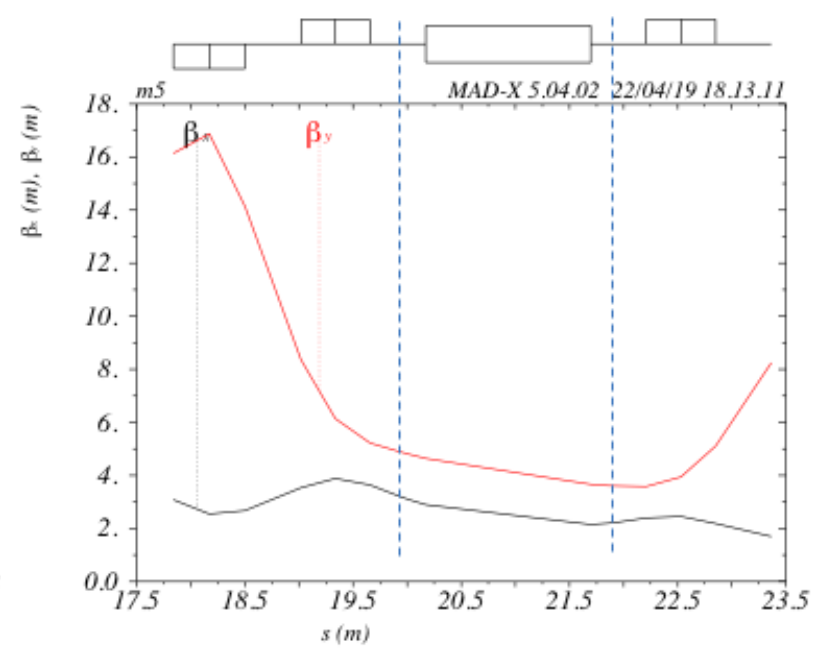

(b) $\beta_{x, y}$

Figure 4.12: The dotted lines in this figure represent the position of the first and second wedges respectively.

If one examines figure $4.12 \mathrm{a}$, it is observed that the beam dispersion at the first wedge position is smaller than the beam dispersion at the second wedge position. This scenario is represented by figure $4.13 \mathrm{a}$. It is noted that in the case of figure $4.13 \mathrm{a}$, the beam enters 


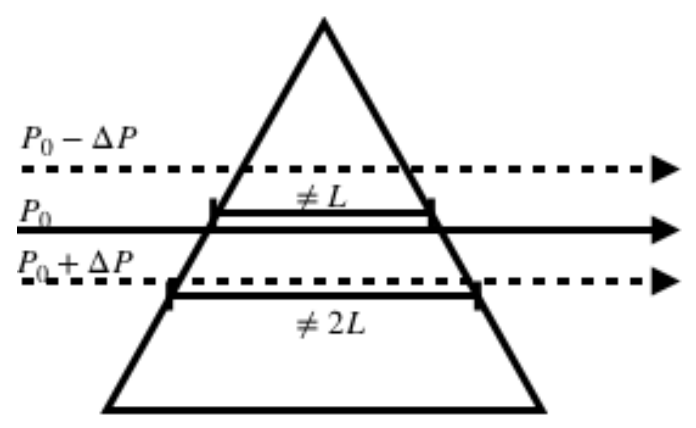

(a) Small Dispersion

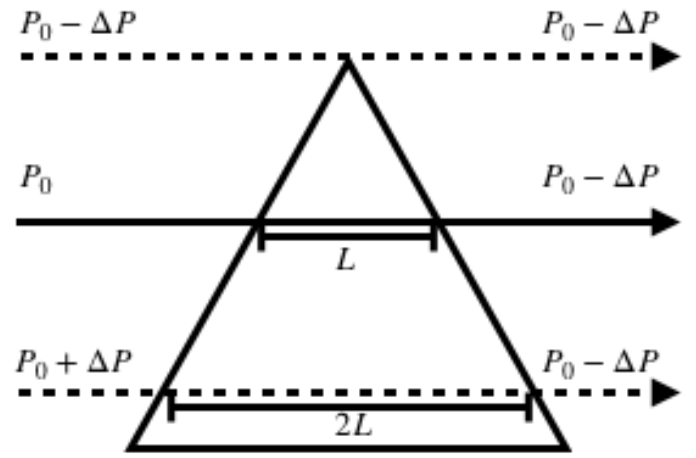

(b) Large Dispersion

Figure 4.13: This figure represents a distribution with small dispersion and a distribution with large dispersion.

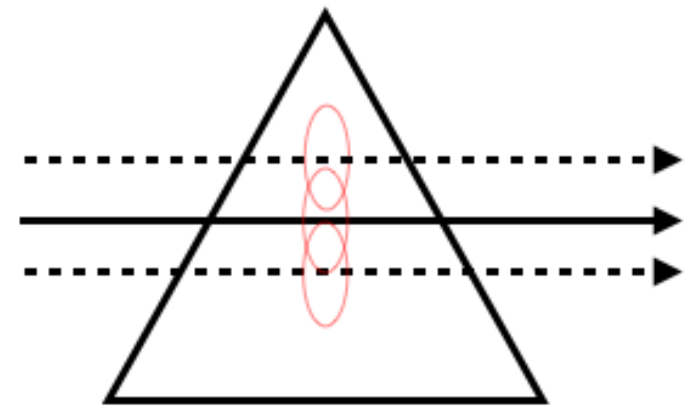

(a) Large $\beta$

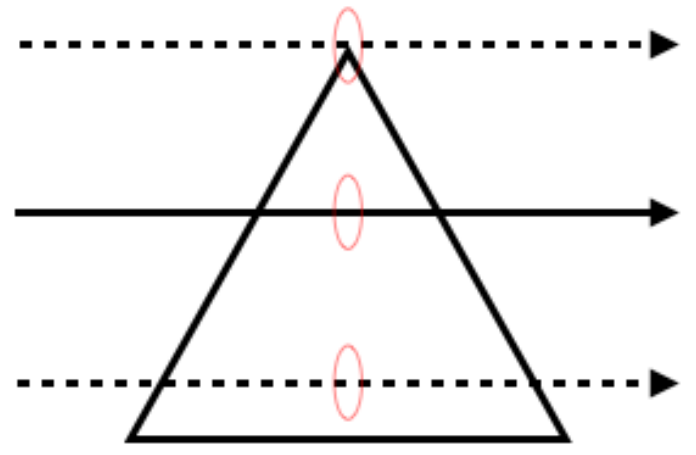

(b) Small $\beta$

Figure 4.14: This figure represents a distribution with large $\beta$ and small $\beta$.

the wedge as a more compact bunch than compared to figure $4.13 \mathrm{~b}$. As a result, the particles with momentum $p-\Delta p$ will receive a momentum reduction. If one looks back to the ideal case of figure 2.2, one notices that the particles with momentum $p-\Delta p$ receive no momentum reduction as they traverse the tip of the wedge. Similarly, the other particles in figure $4.13 \mathrm{a}$ will not traverse the correct length of wedge $L$, as described in Section 2.1.1, to reach the target $p-\Delta p$ momentum. Comparing the two situations, one sees that if a beam enters a wedge with low dispersion, the wedge will not be utilized to it's full potential. This is one possible cause of the first wedge being less effective than the second. 
Now, the difference in the $\beta_{x}$ value at the first and second wedges can be examined as illustrated in figure $4.12 \mathrm{~b}$. The red circles in figure 4.14 represent the possible position of a particle with the corresponding momentum. A larger $\beta$ amplitude will lead to a larger red circle, or a wider range of positions for the particle to be at. In fact, the size of the beam will scale with the square root of $\beta$. It is noted that the size of a beam can be determined by $\sigma=\sqrt{\epsilon \beta}$, where $\sigma$ is the size of the beam. In figure $4.14 \mathrm{a}$ one observes the case of a larger $\beta$ value. It is seen that the larger red circles overlap, which results in particles of high momentum traversing a thinner part of the wedge than intended and vice versa.

From observing the $\beta$ and $D$ values at wedge one and wedge two, one can come to the conclusion that a smaller $\beta$ value and larger $D$ correlate to a more effective wedge. In other words, the larger the ratio of $D / \sqrt{\beta}$ at the wedge - that is, the larger the dispersed beam size is relative to the beam size in the absence of dispersion - the more effective a wedge will be. $\sqrt{\beta}$ goes as a square root because the beam size goes as $\sigma=\sqrt{\epsilon \beta}$. If the $D_{\mathrm{x}}$ and $\beta_{x}$ values are examined at the first wedge, they are about 0.76 and 3.2 respectively. Examining these same values at the second wedge one finds values of 0.85 and 2.2 respectively. If the ratio of $D / \sqrt{\beta}$ is compared at each wedge position, it is found that the ratio at the second wedge is about 1.35 times larger than the ratio at the first wedge. If one looks back to figure 4.9 , one sees that the second wedge is about 1.16 times more effective than the first wedge when they're both placed $6 \mathrm{~mm}$ into the beam path. Comparing the ratio $D / \sqrt{\beta}$ between wedge one and wedge two and the ratio of performance in figure 4.9 , one concludes that a larger value of $D / \sqrt{\beta}$ at a given wedge results in more effective performance out of that wedge.

A future study could be done as to how the beam parameters could be changed in order to improve the effectiveness of the first wedge, resulting in even more muons around the target momentum. 


\subsection{Wedge Rotation Simulation}

In this section a study on how rotating each wedge separately affects the amount of particles within the momentum acceptance window at the end of the M5 line will be examined. In this simulation, one wedge's rotation angle will be altered at a time while the other wedge is completely removed from the lattice.

If it is observed that altering the wedge's rotation angles can provide more particles within the momentum acceptance window at the end of the M5 line, perhaps an argument can be made to alter the wedge's rotation angles in the fermilab g-2 experiment.

\subsubsection{Analysis}

In the following section, two different cases for wedge one and wedge two separately will be observed. In the first case, the altering of one wedge's rotation angle while the other wedge is removed will be studied. In this first case, the wedge has been left in it's original position, meaning that the wedge tip is at the presumed beam center and the rotation angle is 0 degrees. In case two, the same analysis as case one will be performed, except this time the wedge will be inserted $6 \mathrm{~mm}$ into the beamline. inserting the wedge $6 \mathrm{~mm}$ into the beamline allows one to study the effects of changing the wedge rotation angle when the wedge is at an ideal position as described in Section 4.6.

These two cases will be repeated for both wedge one and wedge two. The wedge will be given the freedom to rotate 16 degrees in each direction as shown in figure 4.15 . Rotating beyond 16 degrees in either of the two directions becomes mechanically unrealistic in the fermilab g-2 experiment. 


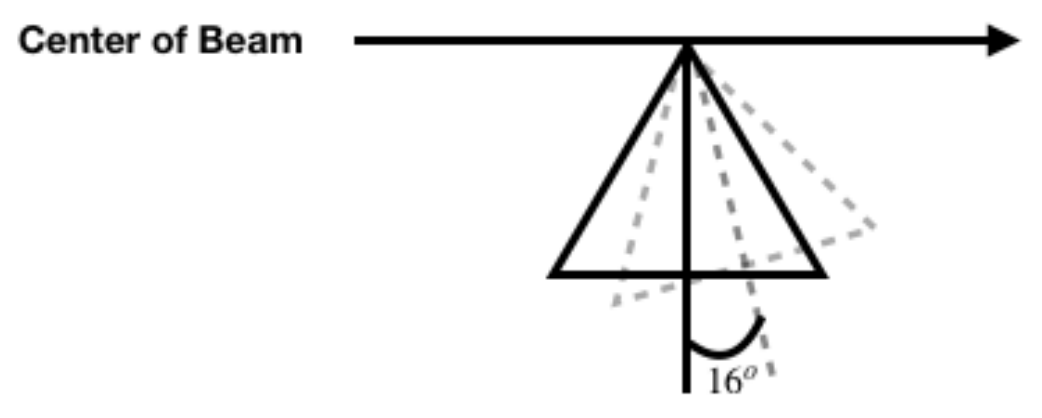

Figure 4.15

\subsubsection{Results}

First, cases one and two where only wedge one's rotation angle is altered will be examined.

In figure 4.16 one observes that altering the first wedge's rotation angle, about 6 degrees in each direction, with the wedge in it's original position leads to a small increase of about $2 \%$, in particles within the momentum acceptance window at the end of M5. However, it is important to notice that if the wedge is placed in it's ideal position $(6 \mathrm{~mm}$ in), as found from Section 4.6, then altering the wedge rotation angle mostly leads to a negative effect on particles within the momentum acceptance window.

Using this information, one may conclude that altering the first wedge's rotation angle does not provide any drastic improvement in particles within the momentum acceptance window at the end of M5. With this in mind, one may now examine case one and two for the second wedge to observe how the rotation angle of wedge two affects particles within the momentum acceptance window at the end of M5.

In figure 4.17, one can observe a similar trend that was observed in figure 4.16. It is seen that with the second wedge in it's original position, one can obtain about an $8 \%$ increase in particles within the momentum acceptance range while changing the wedge 


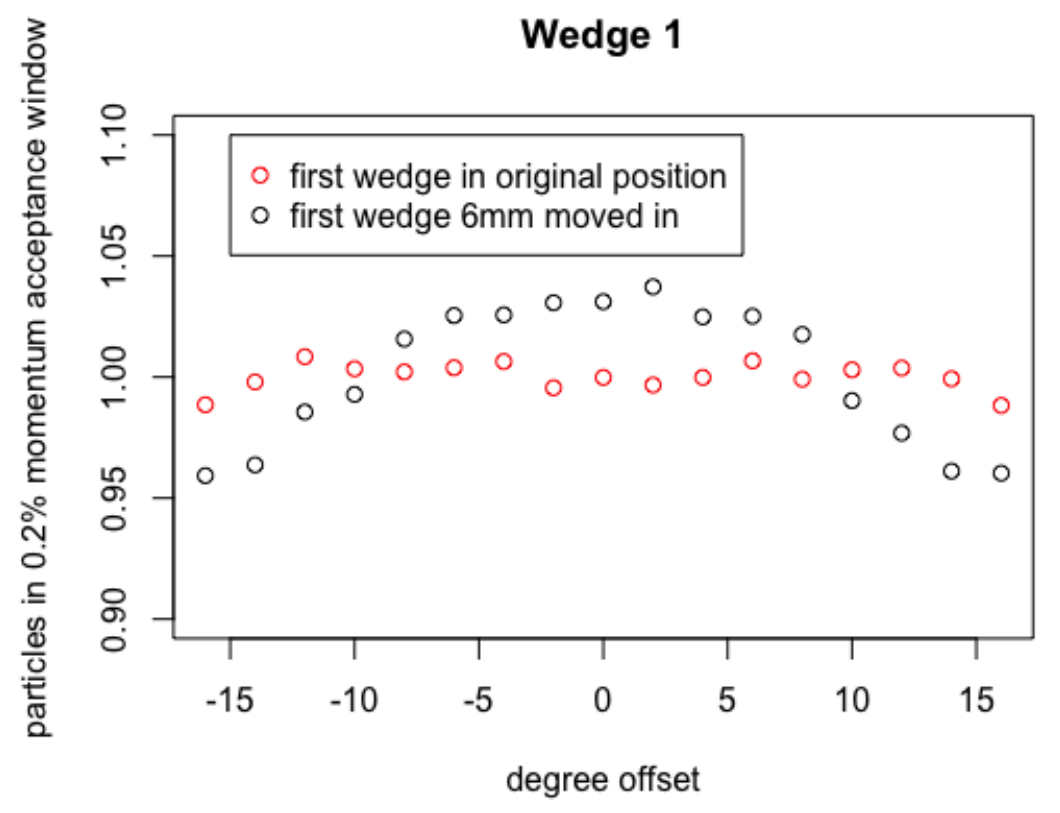

Figure 4.16: Here one observes the effects of altering the first wedge's rotation angle on the amount of particles within the momentum acceptance range at the end of the M5 line. This plot is scaled to the amount of particles within the $0.2 \%$ momentum acceptance window at the end of M5 when wedge 1 is at it's original position and rotation angle, $0 \mathrm{~mm}$ and $0 \mathrm{deg}$ respectively.

rotation angle. However, with the second wedge placed at it's ideal position of $6 \mathrm{~mm}$, altering the wedge only causes a negative effect. A quick comparison of wedge one and wedge two maintains that wedge two is more effective than wedge one in this study as well.

It should be noted that altering each wedge's rotation angle had no significant effects on the emittance at the end of the M5 line when compared to a run where the wedge's rotation angles are left unaltered.

Now that a brief look at the effects of altering the rotation angles of wedge one and wedge two has been completed, one can come to some conclusions. It is shown that with each of the wedges in their original positions, altering the wedge rotation angle can provide a slight increase, $2 \%$ for wedge 1 case 1 and $8 \%$ for wedge 2 case 1 , in particles within 


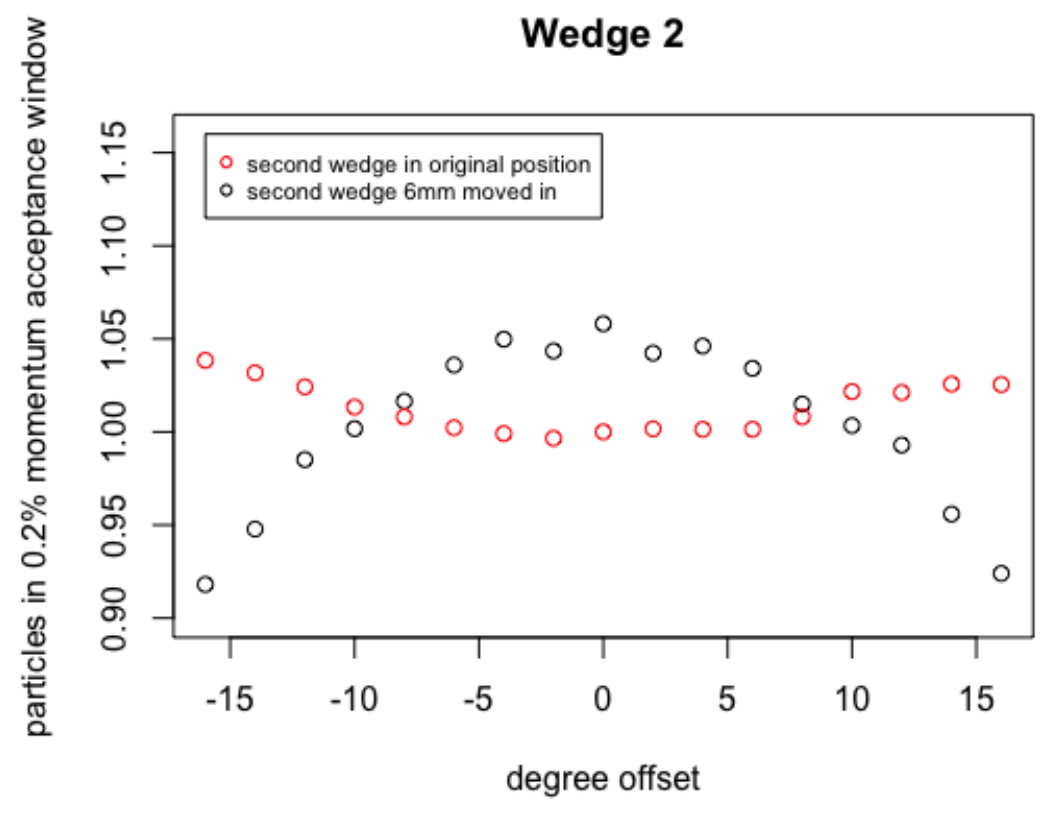

Figure 4.17: Here one observes the effects of altering the second wedge's rotation angle on the amount of particles within the momentum acceptance range at the end of the M5 line. This plot is scaled to the amount of particles within the $0.2 \%$ momentum acceptance window at the end of M5 when wedge 2 is at it's original position and rotation angle, $0 \mathrm{~mm}$ and $0 \mathrm{deg}$ respectively.

the momentum acceptance window at the end of the M5 line. It is also observed that with each wedge placed $6 \mathrm{~mm}$ into the beam path, altering the wedge rotation angle only provides a negative effect on particles within the momentum acceptance range. One can conclude that altering the wedge rotation angle does not provide as much improvement as altering the position of the wedge as seen in Section 4.6.

A future study could be conducted to observe the effects of having both wedges placed in the lattice at the same time and altering both wedge's rotation angles simultaneously. Taking this concept further, a study could be done where both wedge's rotation angles and positions are being altered simultaneously. This analysis could provide more insight as to if altering the wedge's rotation angles could prove useful. 


\subsection{MADX Simulation}

At this point, some good information on how to reduce the $P_{\mathrm{STD}}$ and the growth of $\varepsilon_{x, y}$ has been gathered. The next step is now to observe how the beam parameters change downstream of the wedges. As discussed in Section 2.2, the beam parameters after the wedges such as emittance may have increased to an unstable level and may not be able to traverse the rest of the M5 line without losing some particles to the walls of the beam pipe. In order to observe how the beam parameters act after the wedges, one can turn to MADX.

In this section, two different cases of the beam parameters downstream of the wedges will be examined. The first case will be a case in which there are no wedges placed in the lattice, while the second case will be a case in which both wedges are placed in the lattice. Ideally, one would like to observe minimal changes in the beam parameters between the two cases, especially at the end of the M5 line. If the differences between the two cases is large at the end of the M5 line, this could potentially lead to issues in delivering beam to the storage ring.

\subsubsection{Analysis}

To begin, one can take the beam parameters, from immediately after the second wedge in a g4beamline run where the wedge's are in their unaltered positions, and transfer them to MADX. In this case, the parameters in which are desired to be transfered to MADX are $\beta, \beta^{\prime}, \alpha, \alpha^{\prime}, D$, and $D^{\prime}$. These parameters will serve as the initial conditions for the MADX lattice that begins just after the second wedge and goes until the end of the M5 line. 
It should be noted that the current operational quad strengths are being used for this analysis.

Next, MADX is run for case one and case two. MADX simplifies the analysis by providing the beam parameters of interest, $\beta_{x, y}$ and $D_{x, y}$, at each element throughout the lattice. Plotting the beam parameters downstream of the wedges will allow one to observe the effects of the wedges on the beam parameters.

\subsubsection{Results}

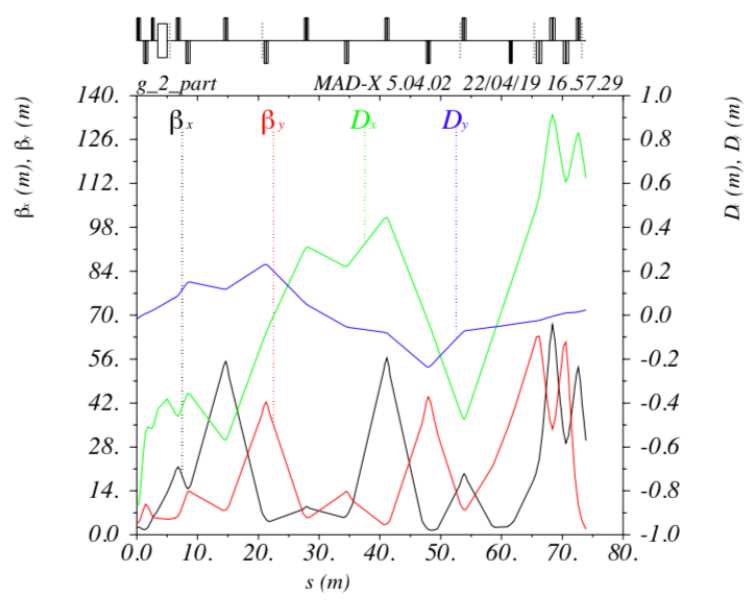

(a) Case 1

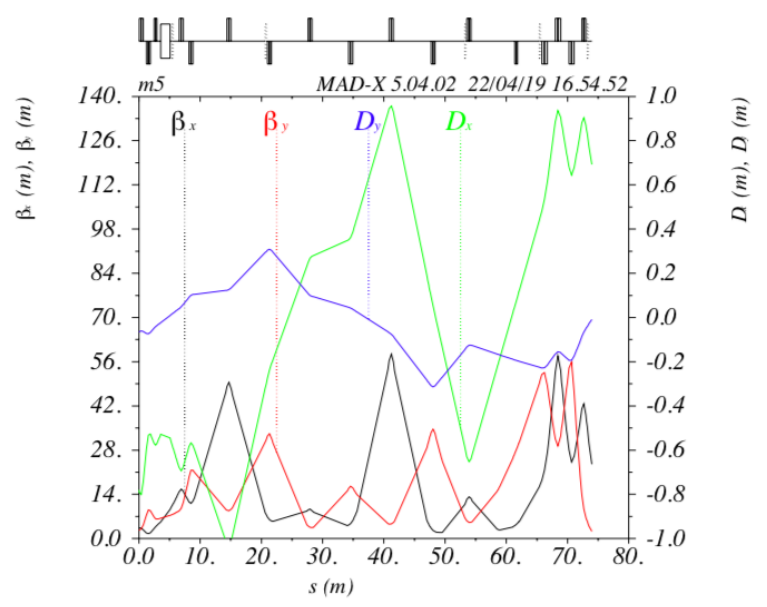

(b) Case 2

Figure 4.18: Figure (a) represents Case 1, where there are no wedges placed in the lattice. Figure (b) represents Case 2, where both wedges are placed in the lattice at their unaltered position and rotation angle. Each of these figures begins immediately after the second wedge and ends at the end of M5.

First, figure 4.18, where case 1 and case 2 are displayed separately will be examined. At first glance it can be difficult to observe the difference in parameters between both of these cases. To get a more intuitive look at the two cases, one can subtract the case 1 parameters from the case 2 parameters. One may now more clearly observe, in figure 4.19, the differences in $\beta_{\mathrm{x}, \mathrm{y}}$ and $D_{\mathrm{x}, \mathrm{y}}$. 


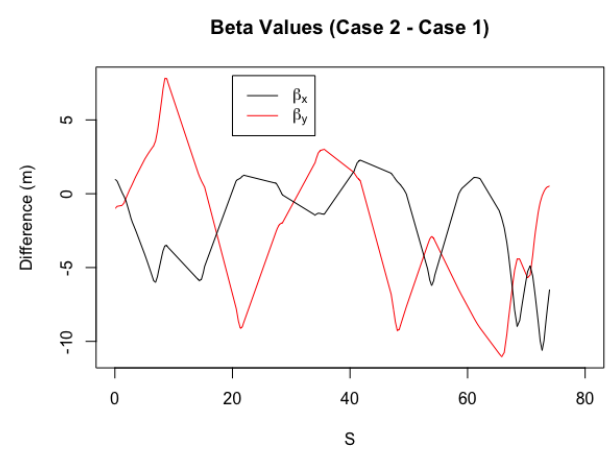

(a) Difference in $\beta_{x, y}$

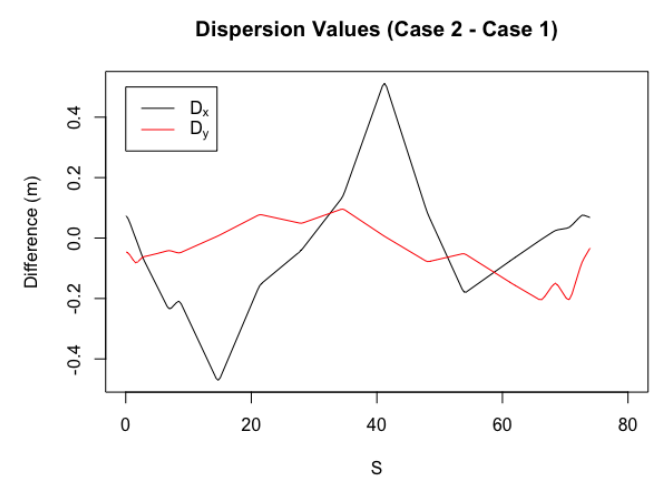

(b) Difference in $D_{x, y}$

Figure 4.19: This figure displays the differences in $\beta_{\mathrm{x}, \mathrm{y}}$ and $D_{\mathrm{x}, \mathrm{y}}$ between Case 1 and Case 2.

From figure 4.19, it is seen that the parameters between the two cases are indeed different. It is noted that at the end of the lattice, all of the parameters, with the exception of $\beta_{x}$, have nearly zero difference between the two cases. Referring to figure 4.14 , it is seen that having a smaller $\beta_{x}$ value will actually prove useful when trying to fit beam into the storage ring as the particles will be on a more tightly bound trajectory. Noting that a smaller $\beta$ value corresponds to a particle on a tighter bound trajectory, on can claim that $\beta_{\mathrm{x}}$ being lower in case 2 than in case 1 will not cause any newfound problems since the particle will be closer to it's design trajectory.

A future, more in depth, study could be done to find the optimal quadrupole strengths downstream of the wedges to deliver the maximum amount of muons to the storage ring. MADX provides many useful tools to aid one in this study, such as the MATCH and VARY commands. The MATCH and VARY commands give one the ability to set restrictions on beam parameters at each element within the lattice. These commands also allow the user the ability to allow elements, such as quadruples, vary their strength as MADX sees fit in order to satisfy the users custom beam parameter constraints. Using these tools, one 
could provide an in depth analysis as to how altering quadrupole strengths may prove useful to an experiment.

\subsection{Effects on Beam Spin}

An important aspect of the g-2 experiment is the muon spin. In order to use wedges in a high precision experiment, one needs to make sure that the spin of each muon is not affected while traversing the wedges. If traversing the wedges causes the beam spin distribution to become more dispersed, this could lead to difficulty in measuring the anomalous MDM inside the storage ring downstream.

\subsubsection{Analysis}

To analyze the beam spin, one can once again utilize the virtual detector immediately after the second wedge in the G4beamline lattice. In this analysis, how the spin changes in each transverse coordinate after the beam has traversed the pair of wedges will be examined. If the spin angles after the second wedge differ in this simulation when compared to a simulation without wedges in place, as shown in figure 4.20 , one would have to reconsider the approach to using wedges.

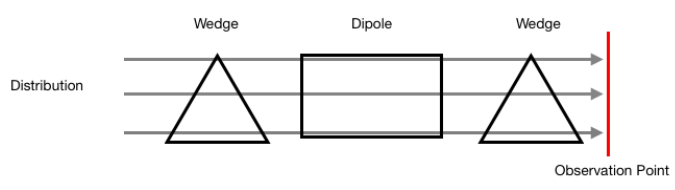

(a)

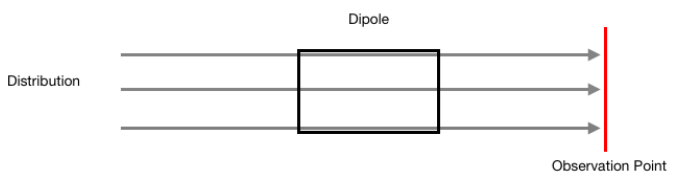

(b)

Figure 4.20: One simulation will be run with wedges in the lattice, (a), and compared to a run with the wedges removed from the lattice, (b). The observation point is where the spin in each of these two cases will be examined. 
As G4beamline provides spin vector components $S_{x}, S_{y}$ and $S_{z}$ (called PolX, PolY, and PolZ in the code output), the direction angles from these components will be calculated. Calculating the direction angles allows one to compare how the spin directions differ, at a observation point after the second wedge, in a run with wedges in place compared to a run without wedges.

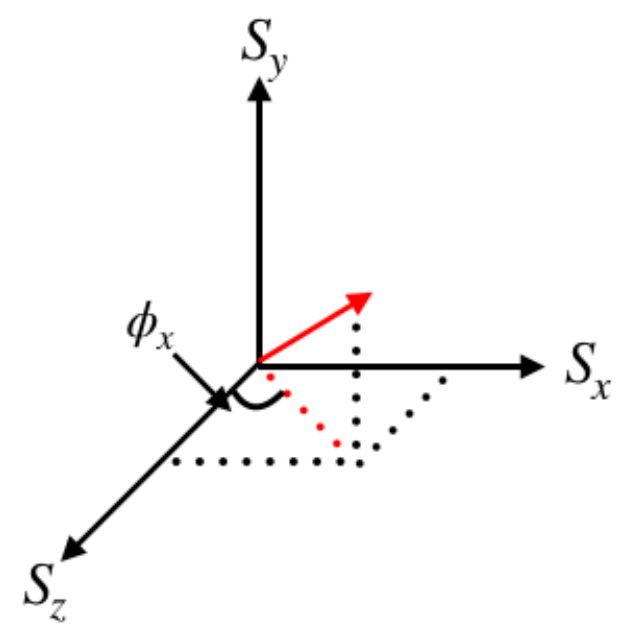

(a)

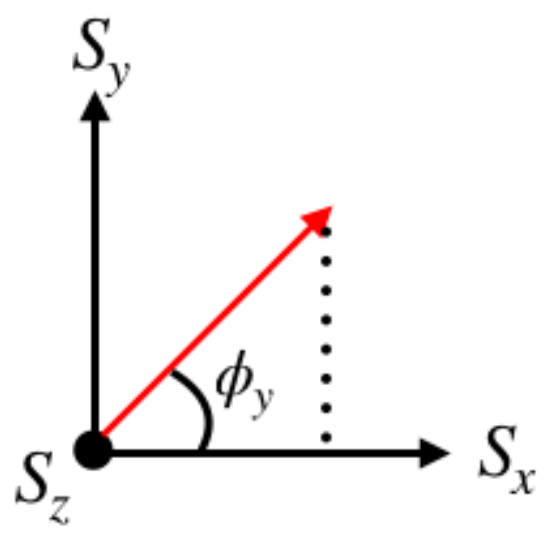

(b)

Figure 4.21: This figure shows how $\phi_{x}$ and $\phi_{y}$ are defined.

From figure 4.21 one sees that

$\tan \phi_{x}=S_{x} / S_{z}$

and

$\tan \phi_{y}=S_{y} / \sqrt{1-S_{y}^{2}}$.

So, for each particle in the distribution, $\phi_{x}$ and $\phi_{y}$ are computed from the polarization components; one can then compare these spin angles, for each case of 4.20 , at the observation point after the wedges by computing the difference. The results are plotted in figure 4.22 . 


\subsubsection{Results}

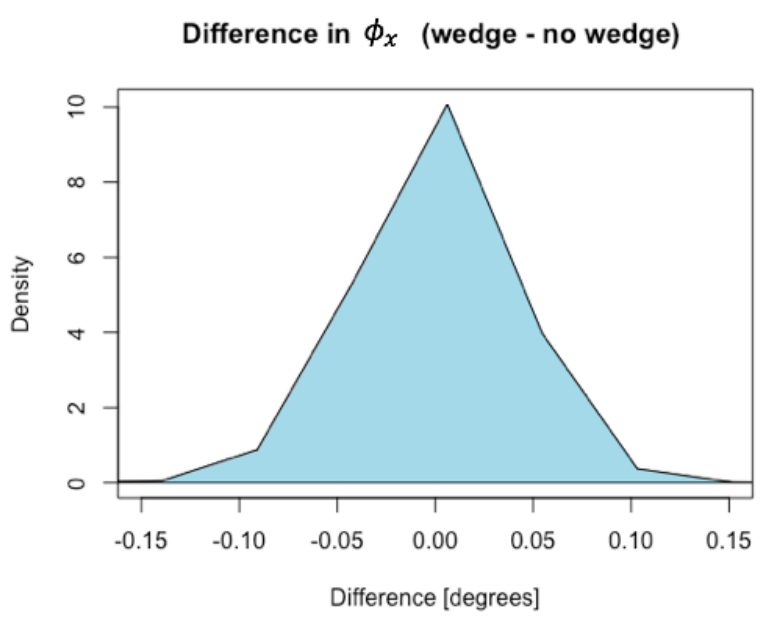

(a)

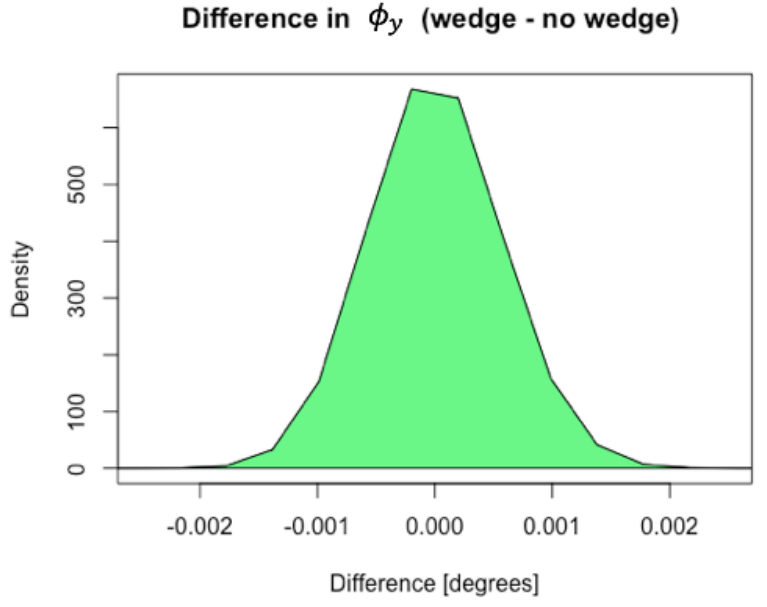

(b)

Figure 4.22: This figure shows the differences in $\phi_{x}$ and $\phi_{y}$ for a simulation with wedges in the lattice compared to a simulation with the wedges removed from the lattice.

In figure 4.22 , one observes the differences in $\phi_{x}$ and $\phi_{y}$ between a simulation with wedges and a simulation without wedges using 6000 particles. It is noted that the difference in $\phi_{x}$ is on the order of $10^{-1}$ degrees or less, while the difference in $\phi_{y}$ is one the order of $10^{-3}$ degrees or less.

The mean value of $\phi_{x}$ in our simulation with the wedges in the lattice is 47.15790 degrees while the $\phi_{x}$ value without the wedges in the lattice is 47.15792 degrees. The difference of $2 \times 10^{-5}$ degrees is insignificant in our simulation of 6000 particles and indicates that the spin distribution is not affected when passing through the wedges. The vertical spin direction similarly is unaffected, as it's mean changes by half as much as above. It is also noted that the standard deviation in $\phi_{x}$ in simulations with and without wedges in the lattice are 14.9242 and 14.9243 degrees respectively. Thus, the spread in 
the spin angle distributions is also unaffected. Likewise, the spread in spin angles for the vertical direction is also unaffected. 


\section{CHAPTER 5}

\section{CONCLUSIONS}

The aim of all of the analysis in chapter 4 was to provide evidence supporting the effectiveness of the two wedges in a high precision experiment. The main objective using these two wedges was to reduce the $P_{\mathrm{STD}}$ in order to deliver more muons to the end of the M5 line within the momentum acceptance window. In this analysis we've leveraged G4beamline and MADX to show how one can reduce the $P_{\text {STD }}$ as well as recapture the beam.

In regards to the reduction of $P_{S T D}$, it is observed that the two wedges do indeed prove to be an asset. With the wedges placed in the lattice at an unaltered position, one observes a decrease in $P_{\text {STD }}$ by $28 \%$, which leads to an increase of $28 \%$ in the amount of particles within the momentum acceptance window at the end of M5 when compared to a run with no wedges. With the wedges placed in the lattice, and through altering the wedges position, a reduction in $P_{\text {STD }}$ of only about $1.3 \%$ is observed which has led to an increase in muons delivered to the end of the M5 line within the momentum acceptance window by $10 \%$ when compared to a run with the wedges in their original position.

We've also showed through simulation, in Section 4.7, that altering the wedge angle may not be worth while as it can lead to a reduction, of about $5-10 \%$, in the amount of muons within the momentum acceptance window if the wedges position is also being altered.

In order to get these reductions in $P_{\mathrm{STD}}$, we've had to forfeit an increase in emittance after the wedges.Through simulation in Section 4.5, we've been able to show that modifying the beam parameters before the wedges can be helpful when attempting to reduce 
the emittance growth downstream of the wedges. It is observed, in figure 4.6 (d) and (c), that one is able to reduce the emittance growth in the $x$ and $y$ directions after the wedges by about $8 \%$ and $30 \%$ respectively when compared to the base distributions emittance growth. The base distribution was the distribution obtained by running G4beamline using the lattice described in the TDR [11]. In order to obtain these improvements, one must be able to change $\beta^{*}$ and $\beta^{*}$ from 3 and 7.3 to 2 and 2 respectively. Changing the values of $\beta^{*}$ and $\beta_{y}^{*}$ corresponds to changing $\beta_{x}$ and $\beta_{y}$ before the wedges from 2.5 and 7.1 to about 2.6 and 2.6. respectively.

We've also been able to show, through G4beamline simulations in Section 4.9, that the wedges will not have any noticeable effect on the spin angles of the muons as they pass through the wedge absorbing material. When comparing the muon spin distributions from a run without wedges to a run with wedges, one observes, in figure 4.22 , a difference on the order of $10^{-1}$ degrees or less for $\phi_{x}$ and $10^{-3}$ degrees or less for $\phi_{y}$.

With these results in mind, it is noted that multiple other studies to be done have been identified that could benefit future experiments. Specifically we've outlined that a study can be done on how to reduce the $\beta_{x, y}$ values before the wedges by altering quadrupoles upstream. Reducing $\beta_{x, y}$ values upstream of the wedges would lead to a decrease in emittance growth downstream of the wedges. We've also pointed out that through the use of MADX, one can find ideal quadrupole strengths downstream of the wedges to recapture the beam in the most efficient way possible. Finally, another important study that could be done is a study on how altering the wedge angles/positions simultaneously can affect the amount of particles within the momentum acceptance window at the end of M5. Understanding these outlined studies will be important in increasing the efficiency of future experiments. 


\section{REFERENCES}

[1] Bennett, G.W, et al. Measurement of the Negative Muon Anomalous Magnetic Moment to 0.7 Ppm, 21 Feb. 2004, www.g-2.bnl.gov/hepex0401008.pdf.

[2] Chislett, Rebecca. "The Muon EDM in the g-2 Experiment at Fermilab." EPJ Web of Conferences, vol. 118, 2016, p. 01005., doi:10.1051/epjconf/201611801005.

[3] Edwards, Donald A., and Michael J. Syphers. An introduction to the physics of high energy accelerators. John Wiley and Sons, 2008.

[4] Syphers, Mike, and Elvin Harms. "Fundamentals of Accelerator Physics and Technology." Northern Illinois Center for Accelerator and Detector Development, USPAS, 15 Jan. 2018, nicadd.niu.edu/syphers/uspas/2018w/some-notes-onellipses.html

[5] Goldstein, Herbert, et al. Classical Mechanics. 3rd ed., Addison-Wesley, 1981.

[6] Particle Data Group. "Particle Data Group - 2018 Review.” Particle Data Group 2018 Review, pdg.lbl.gov/.

[7] Khan, Faiz M., and John P. Gibbons. Khans the Physics of Radiation Therapy. 3rd ed., Wolters Kluwer, 2016.

[8] "Passage of Particles Through Matter", http://pdg.lbl.gov/2018/reviews/rpp2018rev-passage-particles-matter.pdf.

[9] Bradley, J., et al. "International Particle Accelerator Conference." Initial Studies into Longitudinal Ionization Cooling for the Muon g-2 Experiment. 
[10] Syphers, Michael. “G Minus 2 Project Document 2343-v4.” GM2, 13 Nov. 2014, gm2docdb.fnal.gov/cgi-bin/ShowDocument?docid=2343.

[11] J. Grange et al. [Muon g-2 Collaboration], "Muon (g-2) Technical Design Report," arXiv:1501.06858 [physics.ins-det].

[12] Rogers, C. T., et al. Wedge Absorber Design for the Muon Ionisation Cooling Experiment. No. MICE-NOTE-290. 2010.

[13] T. Roberts. G4beamline Version 3.02.1 [Computer software], 2004. Accessed from: http://g4beamline.muonsinc.com

[14] European Organization for Nuclear Research . MADx (Methodical Accel- erator Design) Version 5.02.00. [Computer software], 1990. Accessed from: http://madx.web.cern.ch/madx

[15] R Core Team. R: A language and environment for statistical computing [Computer software]. R Foundation for Statistical Computing, Vienna, Austria, 2013. Accessed from: http://www.R-project.org/

[16] Stratakis, D., et al. "Performance analysis for the new G-2 experiment." arXiv preprint arXiv:1607.00303 (2016).

[17] Stratakis, Diktys, et al. "Commissioning and First Results of the Fermilab Muon Campus.” Physical Review Accelerators and Beams, vol. 22, no. 1, Jan. 2019, pp. 1-13., doi:10.1103/physrevaccelbeams.22.011001.

[18] “National Energy Research Scientific Computing Center.” National Energy Research Scientific Computing Center, www.nersc.gov/.

[19] M.J. Syphers, "Storage Rings for Precision Physics Applications", notes for course given at US Part. Accel. School, Winter 2019, Appendix D. 
[20] V. Bargmann, L. Michel, and V. L. Telegdi, "Precession of the Polarization of Particles Moving in a Homogeneous Electromagnetic Field”, Phys. Rev. Lett. 2, 435 (1959). 